\title{
DNA methylation profiling in peripheral lung tissues of smokers and patients with COPD
}

\author{
Isaac K. Sundar ${ }^{1 *}$, Qiangzong Yin ${ }^{1}$, Brian S. Baier ${ }^{1}$, Li Yan ${ }^{3}$, Witold Mazur ${ }^{4}$, Dongmei Li ${ }^{2}$, Martha Susiarjo \\ and Irfan Rahman'
}

\begin{abstract}
Background: Epigenetics changes have been shown to be affected by cigarette smoking. Cigarette smoke (CS)-mediated DNA methylation can potentially affect several cellular and pathophysiological processes, acute exacerbations, and comorbidity in the lungs of patients with chronic obstructive pulmonary disease (COPD). We sought to determine whether genome-wide lung DNA methylation profiles of smokers and patients with COPD were significantly different from non-smokers. We isolated DNA from parenchymal lung tissues of patients including eight lifelong non-smokers, eight current smokers, and eight patients with COPD and analyzed the samples using Illumina's Infinium HumanMethylation450 BeadChip.

Results: Our data revealed that the differentially methylated genes were related to top canonical pathways (e.g., G beta gamma signaling, mechanisms of cancer, and nNOS signaling in neurons), disease and disorders (organismal injury and abnormalities, cancer, and respiratory disease), and molecular and cellular functions (cell death and survival, cellular assembly and organization, cellular function and maintenance) in patients with COPD. The genome-wide DNA methylation analysis identified suggestive genes, such as NOSTAP, TNFAIP2, BID, GABRB1, ATXN7, and THOC7 with DNA methylation changes in COPD lung tissues that were further validated by pyrosequencing. Pyrosequencing validation confirmed hyper-methylation in smokers and patients with COPD as compared to non-smokers. However, we did not detect significant differences in DNA methylation for TNFAIP2, ATXN7, and THOC7 genes in smokers and COPD groups despite the changes observed in the genome-wide analysis.
\end{abstract}

Conclusions: Our study suggests that DNA methylation in suggestive genes, such as NOS1AP, BID, and GABRB1 may be used as epigenetic signatures in smokers and patients with COPD if the same is validated in a larger cohort. Future studies are required to correlate DNA methylation status with transcriptomics of selective genes identified in this study and elucidate their role and involvement in the progression of COPD and its exacerbations.

Keywords: DNA methylation, Lung, Epigenetics, COPD, Smokers, Pyrosequencing

\section{Background}

Cigarette smoking is the main etiological factor in the pathogenesis of chronic obstructive pulmonary disease (COPD). Several mechanisms have been proposed in the pathogenesis of COPD, such as oxidative stress, inflammation, protease/antiprotease, epigenetics, apoptosis, and cellular senescence [1]. Cigarette smoke (CS) is known to

\footnotetext{
* Correspondence: isaac_sundar@urmc.rochester.edu

${ }^{1}$ Department of Environmental Medicine, University of Rochester Medical

Center, Box 850, 601 Elmwood Avenue, Rochester 14642, NY, USA

Full list of author information is available at the end of the article
}

affect the transcriptional regulation of upstream and downstream target genes involved in different canonical pathways that are implicated in the progression of COPD. However, not all the alterations in the pathogenesis of COPD, e.g., steroid resistance, acute exacerbations, and comorbidity, can be explained by transcriptional changes or cellular abnormalities alone $[2,3]$.

Epigenetic mechanisms, specifically methylation status of the DNA at specific CpG sites, are known to play a crucial role in several chronic inflammatory diseases including cancer and aging $[2,3]$. DNA methylation plays 
an important role in transcriptional regulation, e.g., gene silencing [1,2]. Environmental and genetic factors, such as CS exposure, diet, genetic variation, and aging, trigger oxidative stress that can affect the promoter CpG methylation by recruiting methyl $\mathrm{CpG}$ binding protein 2 and DNA methyltransferases onto various promoters $[1,2]$. Alteration in the methylation status of the promoter affects expression of tumor suppressor, oncogenes, and pro- and anti-inflammatory genes [2]. Previously, studies were conducted using sputum, whole blood, peripheral blood leukocytes, WBCs, alveolar macrophages, small airway epithelium, lung tissues, including buccal brushings, and bronchial brushings of small airways to investigate DNA methylation sites associated with smokers and patients with COPD [4-15]. Different methods of DNA methylation profiling were conducted to assess the epigenetic disruption in key genes and canonical pathways associated with smoking history and COPD status [5-15]. However, the DNA methylation profiling in parenchymal lung tissues of smokers and patients with COPD and their relationship between gene-specific DNA methylation, smoking, and COPD disease progression remains unclear.

DNA methylation is a reversible gene regulatory modification which is shown to be altered by tobacco smoke. Current smokers with a gene-specific DNA hyper- or hypo-methylation can be highly susceptible to disease development based on the patterns of genomic DNA methylation profiles. A growing body of evidence suggests that DNA methylation status in gene promoters can be used as a novel epigenetic biomarker for smoking-related chronic lung diseases, such as COPD and its exacerbations as well as lung cancer [10, 16-19]. The effects of differential DNA methylation patterns mediated by tobacco smoke, particularly in lung tissues from healthy lifelong non-smokers in comparison with smokers and patients with COPD, remains unclear. In the present study, we used a genome-wide DNA methylation analysis (Illumina's Infinium 450K BeadChip array) combined with pyrosequencing approaches to identify novel suggestive genes as epigenetic signatures of DNA methylation in lung tissues. This study could offer promise as measures of tobacco smoke exposure or toxicity and ultimately serve as an indicator for the susceptibility, progression, pathogenesis, and exacerbations of smoking-related chronic lung diseases. Some of the results have been reported in the form of an abstract [20].

\section{Methods}

Ethics statement and scientific rigor/reproducibility

The lung tissue specimens from normal, lifelong nonsmokers, smokers, and patients with COPD were collected by the Department of Medicine and Pathology, Helsinki University Central Hospital. The clinical characteristics of the subjects/patients used in this study are summarized (Table 1). We used a rigorous/robust and unbiased approach throughout the experimental plans and during analyzing the data so as to ensure that our data are reproducible along with by full and detailed reporting of both methods and raw/analyzed data. All the key biological and/or chemical resources that are used in this study were validated and authenticated (methods and resources) and are of scientific standard from commercial sources. Our results adhere to NIH standards of reproducibility and scientific rigor.

\section{Human lung tissues}

Lung tissue specimens from 24 subjects/patients including 8 lifelong non-smokers, 8 current smokers with normal lung function, and 8 patients with COPD undergoing resection for suspected lung tumor (either malignant or nonmalignant-local carcinoma or hamartoma) or lung transplantation from the Department of Medicine and Pathology, Helsinki University Hospital as described in our previous study [21, 22]. Tumor-free peripheral lung tissues were immediately stored at $-80{ }^{\circ} \mathrm{C}$ for DNA extraction. The clinical characteristics of the subjects/ patients used in this study are provided (Table 1).

\section{Genomic DNA isolation and DNA methylation profiling}

Lung tissues were collected snap frozen and stored at $-80{ }^{\circ} \mathrm{C}$. Genomic DNA was extracted from lung tissue using the Qiagen DNAeasy kit (Qiagen, Valencia, AC) according to the manufacturer's instructions. Briefly, $1 \mu \mathrm{g}$ genomic DNA was bisulfite-converted using EZ DNA Methylation Kit (Zymo Research, Irvine, CA). The bisulfite-converted DNA samples were assayed using Infinium HumanMethylation450 BeadChip array from

Table 1 General clinical characteristics of non-smokers, smokers, and COPD patients

\begin{tabular}{llll}
\hline Parameters & Non-smokers & Smokers & COPD \\
\hline Number of subjects & 8 & 8 & 8 \\
M:F ratio & $6: 2$ & $7: 1$ & $5: 3$ \\
Male:female (\%) & $(75 \%: 25 \%)$ & $(87.5 \%: 12.5 \%)$ & $(62.5 \%: 37.5 \%)$ \\
Age, year & $62.62 \pm 1.87$ & $61 \pm 2.33$ & $56.1 \pm 2.57$ \\
& $(55-69)$ & $(52-71)$ & $(45-63)$ \\
Smoking, pack years & - & $27.14 \pm 5.96^{* * *}$ & $28.8 \pm 4.32^{* * *}$ \\
& & $(10-50)^{\mathrm{a}}$ & $(10-43)^{\mathrm{a}}$ \\
FEV $\%$ predicted & $100.28 \pm 5.55$ & $88.75 \pm 4.04$ & $23 \pm 4.41^{* * * ; \# \#}$ \\
& $(81-124)^{\mathrm{b}}$ & $(68-102)$ & $(10-43)$ \\
FEV $/$ FVC \% & $0.79 \pm 0.01$ & $0.81 \pm 0.02$ & $0.33 \pm 0.03^{* * * ;}$;\#\# \\
& $(0.73-0.85)^{\mathrm{b}}$ & $(0.75-0.95)$ & $(0.25-0.5)$ \\
\hline
\end{tabular}

${ }^{a}$ No pack years details for one subject in the smokers and COPD groups ${ }^{\mathrm{b}}$ No $\mathrm{FEV}_{1} \%$ predicted (one subject) and $\mathrm{FEV}_{1} / \mathrm{FVC} \%$ values (two subjects) in the non-smokers group

${ }^{* * *} P<0.001$ vs. non-smokers, ${ }^{\# \#} P<0.001$ vs. smokers M:F Male to Female ratio, $F E V_{1}$ forced expiratory volume in $1 \mathrm{~s}$ 
Illumina (San Diego, CA). A total of 485,512 CpG sites were probed for each sample that was processed as per the manufacturer's protocol. Image and data analysis of the BeadChips were performed using the Illumina iScan Reader. The image data is then transferred to Illumina GenomeStudio data processing, validation of assay controls, and report generation using the methylation module. The level of methylation for the interrogated locus is determined by calculating the ratio of the fluorescent signals from methylated vs. unmethylated sites. From the genome-wide DNA methylation data, we identified specific genes based on their top canonical pathways and biological functions by functional network analysis using ingenuity pathway analysis (IPA) that were significantly hyper-methylated in lung tissues of smokers and patients with COPD compared to non-smokers for validation by pyrosequencing.

\section{Statistical approaches for DNA methylation analysis and functional network analysis}

Quality control (QC) was first performed on DNA methylation data by filtering out probes with any of the following conditions: (1) Missing methylation beta value in any of the samples, (2) probes on $\mathrm{X}$ and $\mathrm{Y}$ chromosome, (3) any probes associated with SNP, (4) any probes that cannot unambiguously mapped to the Human Reference Genome, and (5) ensured at least 75\% samples with detection $P$ value $<0.05$ in each of the three groups (Non-smokers, NS; Smokers, S; and COPD, C). After the above QC filtering, we have a total of 276,260 probes. Second, the raw DNA methylation level data (beta values) were normalized using the SWAN normalization method in minfi package [23]. The SWAN method was able to correct the bias introduced by the two types of probes in the Illumina $450 \mathrm{~K}$ platform due to chemistry differences in those probes [24]. The quality-controlled and normalized DNA methylation data from the COPD, smoker, and non-smoker groups conducted at two different time points were then combined together. A low DNA methylation level filtering was applied to the postpreprocessed DNA methylation data to filter out DNA methylation loci with maximum methylation level below 0.15 . The difference in methylation levels between different groups at each remained methylation loci was examined using a general linear model approach after adjusting the time effect in the model using the limma package in R/Bioconductor [25]. The estimates of the group differences were examined using a moderated $t$ statistics with empirical Bayes approach to shrink the standard deviations for obtaining robust estimates. The top candidate genes/probes were selected based on uncorrected $P$ values since none of the CpG sites were significant after adjusting the raw $P$ values using the Benjamini-Hochberg procedure. Although those top candidate gene/probes were selected based on uncorrected $P$ values, they did indicate the observed differences between groups that may likely be true positives and possibly reach statistical significance after multiplicity adjustment given a larger sample size which will be further evaluated in future studies. Linear contrasts within the general linear models were conducted for pairwise comparisons between groups (COPD vs. smoker; COPD vs. non-smoker; smoker vs. non-smoker). Top candidates in each pairwise comparison were selected by test statistics and $P$ values (with unadjusted raw $P$ value $P<0.001$ ) from comparisons. Venn diagrams were used to show the overlap of identified top candidates of methylated loci between different pairwise comparisons. Heatmaps of methylation levels from selected DNA methylation loci were generated to show the differences between groups.

For biological insights into top differential methylation changes in relation to smokers vs. non-smokers and COPD vs. non-smokers, we implemented a functional network analysis. Genes annotated from selected differentially methylated probes (DMPs) $P$ value $<0.001$ were included in the analysis of gene regulation network. We used a core analysis of ingenuity pathway analysis (Ingenuity Systems, Inc., Redwood City, CA, USA). Additionally, statistical analysis of significance for genome-wide DNA methylation analysis represented as box plots and pyrosequencing data analysis included as part of the main figures and supplemental data were calculated using one-way analysis of variance (ANOVA) followed by Tukey's multiple comparisons test using GraphPad Prism 6. The results are shown as the mean \pm SEM unless otherwise indicated. $P<0.05$ is considered as statistically significant.

\section{Pyrosequencing analysis}

Validation of selective gene-specific $\mathrm{CpGs}$ identified from HumanMethylation450 BeadChip analysis was performed using the same genomic DNA used for genomewide methylation analysis as described above. We validated the methylation of five CpG sites (NOS1AP, TNFAIP2, BID, GABRB1, ATXN7, and THOC7) identified by the $450 \mathrm{~K}$ array mentioned above and two other CpG sites [AHRR (cg21161138) and SERPINA1 (cg02181506)] based on prior studies $[11,26]$. When we designed the assay for pyrosequencing of target $\mathrm{CpG}$ sites, we included additional $\mathrm{CpG}$ sites along with the $\mathrm{CpG}$ site identified based on genomewide DNA methylation data for all the selected subset of genes for pyrosequencing. Forward and reverse primers are designed using the Pyromark Assay Design Software. Bisulfite conversion and cleanup of DNA samples were performed by using EpiTect Fast Bisulfite Conversion Kits (Qiagen). DNA was amplified in PCR using the PyroMark PCR kit (Qiagen). Pyrosequencing was performed using the PyroMark Q24 Advanced as per the 
manufacturer's instructions. Using the PyroMark CpG Software 2.0, CpG methylation percentages were calculated based on the height of the $\mathrm{T}$ and $\mathrm{C}$ peaks at the methylation site and applying the formula $(\mathrm{C} / \mathrm{C}+\mathrm{T}) \times$ 100. The forward and reverse PCR primers and sequencing primer for specific CpGs are listed (Table 8).

\section{Results}

Genome-wide DNA methylation data analysis was performed using lung tissues from eight patients with COPD (GOLD stages: III-IV) with mean forced expiratory volume in $1 \mathrm{~s}, \mathrm{FEV}_{1}$ predicted $(23 \pm 4.41)$, eight smokers with $\mathrm{FEV}_{1}$ predicted $(88.75 \pm 4.04)$ and eight non-smokers (controls) with normal spirometry (Table 1). There were no significant differences between non-smokers, smokers, and patients with COPD based on their sex and age. All the smokers and patients with COPD had higher pack years of cigarettes smoked compared to non-smokers $(P<0.001)$.

\section{Differential methylation}

We hypothesized that genome-wide lung DNA methylation profiles of smokers and patients with COPD would be significantly different from non-smokers (controls). Boxplot was generated using the boxplot function in $R$. Boxplot was used to describe the distribution of preprocessed DNA methylation level ( $\beta$ values). The central 50 percentile of the $\beta$ values range from 0.35 to 0.90 . The spread of the $\beta$ values were slightly varied within each group. No obvious differences between groups were observed for distribution of $\beta$ values (Additional file 1: Figure S1). Differentially methylated probe (DMP) analysis revealed a total of $10 \mathrm{CpG}$ sites that were possibly differentially methylated between smokers vs. nonsmokers, a total of $280 \mathrm{CpG}$ sites that were possibly differentially methylated between COPD vs. non-smokers, and a total of $10 \mathrm{CpG}$ sites that were possibly differentially methylated between COPD vs. smokers (unadjusted raw $P$ value $P<0.001$ ) (Additional file 2: Table S1, Additional file 3: Table S2 and Additional file 4: Table S3). Similarly, DMP analysis revealed 115 CpG sites (smokers vs. nonsmokers), 1961 CpG sites (COPD vs. non-smokers), and $136 \mathrm{CpG}$ sites (COPD vs. smokers) (unadjusted raw $P$ value $P<0.01$ ) (Additional file 5: Table S4, Additional file 6: Table S5 and Additional file 7: Table S6). Finally, DMP analysis with unadjusted raw $P$ value $P<0.05$ revealed a total of $10,363 \mathrm{CpG}$ sites in smokers vs. non-smokers, a total of $34,151 \mathrm{CpG}$ sites in COPD vs. non-smokers, and a total of 11,339 CpG sites that were differentially methylated between COPD vs. smokers (Additional file 8: Table S7, Additional file 9: Table S8 and Additional file 10: Table S9).

Manhattan plot was generated using the Manhattan. plot function in $R$. Manhattan plot showed the negative
$P$ values on $\log 10$ scale on each chromosome with relatively small unadjusted raw $P$ values corresponding to larger- $\log 10$ ( $p$ value). The chromosome-wide distribution of CpG sites and their comparison groups (nonsmokers vs. smokers, non-smokers vs. COPD, and COPD vs. smokers) were shown (Fig. 1a-c and Tables 2, 3, and 4). Similarly, volcano plot was generated using the volcano plot function in limma package in $R$. Volcano plot is a scatter plot of $\log$ odds ratios vs. $\log$ fold change. The top $10 \mathrm{CpGs}$ sites and associated genes including others $\mathrm{CpG}$ sites that were chosen for validation by pyrosequencing (NOS1AP, TNFAIP2, BID, GABRB1, ATXN7, and THOC7) based on their biological function with corresponding DNA methylation loci that were differentially methylated between different comparison groups are shown in the volcano plots (Fig. 2a-c and Tables 2, 3, and 4). Venn diagram was generated using the Venn diagram function in limma package in $R$. Venn diagram shows the overlap of DNA methylation loci that are shared between different comparisons (Additional file 1: Figure S2). The total number of overlapping genes and probes under each comparison groups shown in Venn diagram were not the same since a few of these did not annotate to genes (Additional file 2: Table S1, Additional file 3: Table S2 and Additional file 4: Table S3). Heatmaps were generated using the heatmap. 2 function in the gplot package in $R$. We have included the DNA methylation loci and samples groups (NS, non-smokers; S, smokers, and C, COPD) for cluster analysis using the hierarchical clustering method. The green color in the heatmap denotes hyper-methylated loci, and the red color in the heatmap denotes the hypo-methylated loci. The sample groups are denoted by different colors. The top 100 genes along with the genes chosen for validation by pyrosequencing were included as part of the cluster analysis in smoker vs. non-smokers (Fig. 3a), COPD vs. nonsmokers (Fig. 3b), and COPD vs. smokers (Fig. 3c), including the list of DMPs (Additional file 11: Table S10, Additional file 12: Table S11 and Additional file 13: Table S12).

From genome-wide methylation data, when we compared possible differentially methylated probes and their associated genes between smokers vs. non-smokers, results revealed the top $10 \mathrm{CpG}$ sites such as ANKRD9 (cg14531093), HLA-DRB5 (cg15011943), TNFAIP2 (cg18620571), ANGPT1 (cg22837763), FAM8A1 (cg10154826), cg17289692, ATXN7 and THOC7 (cg07753241), CNOT1 (cg01096617), ACTN4 (cg25383568), and LOC387646 (cg20377766) (Fig. 4 and Table 2). Similarly, when we compared possible differentially methylated probes and their associated genes between COPD vs. non-smokers, results revealed the top 10 CpG sites such as ANGPT1 (cg22837763), PLD1 


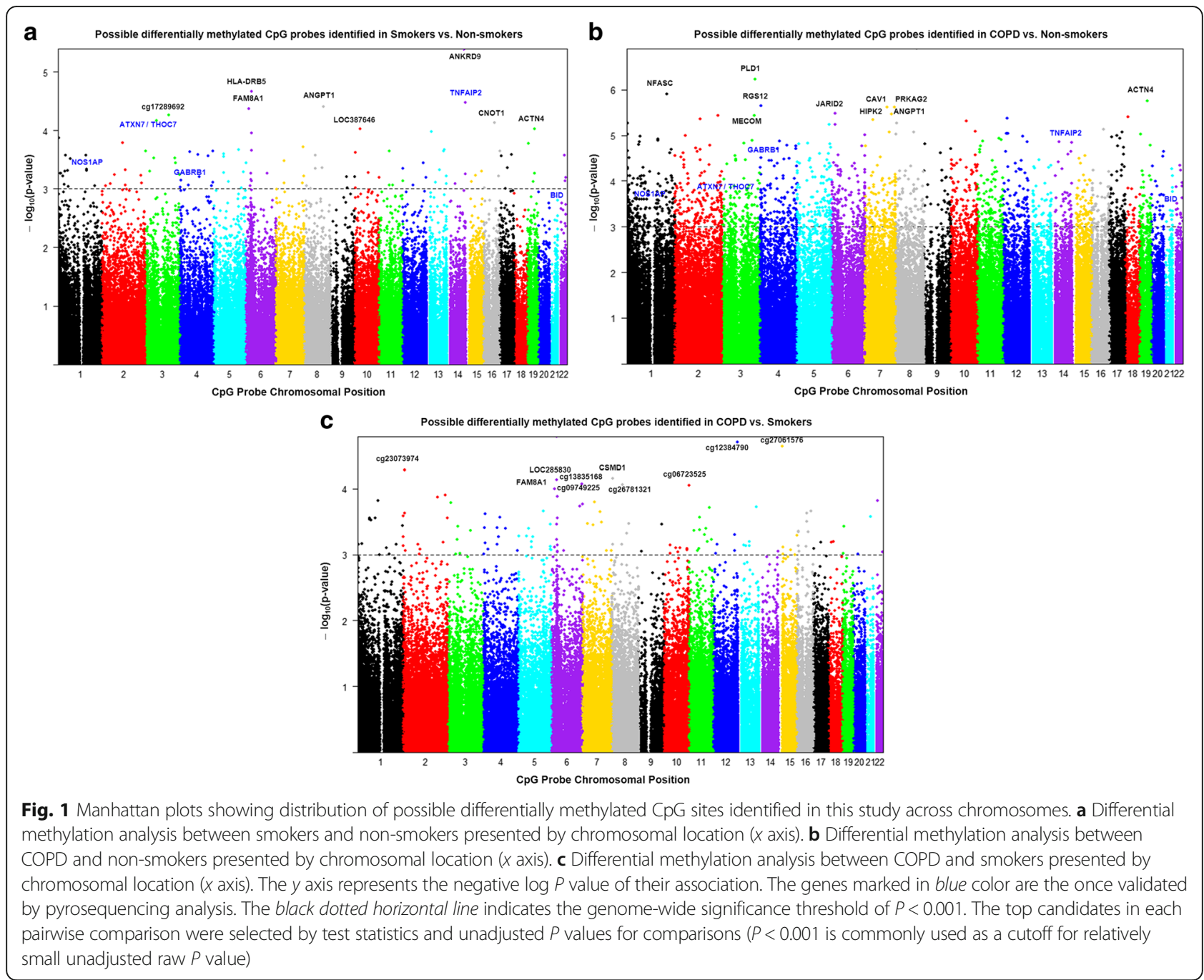

Table 2 Top 10 differentially methylated CpG sites in smokers compared to non-smokers, sorted by $P$ value

\begin{tabular}{|c|c|c|c|c|c|c|}
\hline CpG site & Gene symbol & Chromosome & ${ }^{\text {aProbe Type }}$ & $P$ value & ${ }^{\mathrm{b}}$ Gene location of first annotated transcript & Island location \\
\hline $\operatorname{cg} 14531093$ & ANKRD9 & 14 & 1 & 4.04E-06 & Body & Island \\
\hline cg15011943 & HLA-DRB5 & 6 & $\|$ & $2.16 \mathrm{E}-05$ & Body & S_Shelf \\
\hline cg18620571 & TNFAIP2 & 14 & । & $3.28 \mathrm{E}-05$ & Body & Island \\
\hline $\operatorname{cg} 22837763$ & ANGPT1 & 8 & $\|$ & $3.86 \mathrm{E}-05$ & Body & \\
\hline cg10154826 & FAM8A1 & 6 & $\|$ & 4.26E-05 & $1^{\text {st }}$ Exon & Island \\
\hline cg17289692 & & 3 & $\|$ & $5.48 \mathrm{E}-05$ & & \\
\hline cg07753241 & ATXN7;THOC7 & 3 & $\|$ & $6.74 \mathrm{E}-05$ & TSS1500 & Island \\
\hline cg01096617 & CNOT1 & 16 & $\|$ & $7.50 \mathrm{E}-05$ & Body & \\
\hline cg25383568 & ACTN4 & 19 & I & $9.46 \mathrm{E}-05$ & Body & Island \\
\hline cg20377766 & LOC387646 & 10 & $\|$ & 9.63E-05 & TSS1500 & Island \\
\hline
\end{tabular}

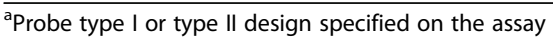

bocation relative to the first listed transcript

${ }^{\mathrm{c}} \mathrm{S}_{\text {_ }}$ denote the downstream end of the island region 
Table 3 Top 10 differentially methylated CpG sites in COPD compared to non-smokers, sorted by $P$ value

\begin{tabular}{lllllll}
\hline CpG site & Gene symbol & Chromosome & aProbe Type & $P$ value & bGene location of first annotated transcript & Island location \\
\hline cg05275153 & RGS12 & 4 & I & $2.24 \mathrm{E}-06$ & Body \\
cg06746365 & PRKAG2 & 7 & $\|$ & $2.48 \mathrm{E}-06$ & Body \\
$\operatorname{cg} 08830492$ & NFASC & 1 & I & $1.24 \mathrm{E}-06$ & $5 \mathrm{UTR}$ \\
$\operatorname{cg} 14630106$ & HIPK2 & 7 & $\|$ & $3.35 \mathrm{E}-06$ & Body \\
$\operatorname{cg} 15558717$ & CAV1 & 7 & $\|$ & $2.40 \mathrm{E}-06$ & Body \\
$\operatorname{cg} 22837763$ & ANGPT1 & 8 & $\|$ & $1.30 \mathrm{E}-07$ & Body \\
$\operatorname{cg} 22920586$ & PLD1 & 3 & $\|$ & $5.93 \mathrm{E}-07$ & 5 UTR \\
$\operatorname{cg} 24408769$ & JARID2 & 6 & I & $3.30 \mathrm{E}-06$ & Body \\
$\operatorname{cg} 25010400$ & MECOM & 3 & $\|$ & $3.64 \mathrm{E}-06$ & Body \\
$\operatorname{cg} 25383568$ & ACTN4 & 19 & I & $1.75 \mathrm{E}-06$ & Body & Island \\
\hline
\end{tabular}

a Probe type I or type II design specified on the assay

${ }^{\mathrm{b}}$ Location relative to the first listed transcript

(cg22920586), NFASC (cg08830492), ACTN4 (cg25383568), RGS12 (cg05275153), CAV1 (cg15558717), PRKAG2 (cg06746365), JARID2 (cg24408769), HIPK2 (cg14630106), and MECOM (cg25010400) (Fig. 5 and Table 3). Finally, when we compared possible differentially methylated probes and their associated genes between COPD vs. smokers, results revealed of the top $10 \mathrm{CpG}$ sites 3 were associated with genes CSMD1 (cg08236285), LOC285830 (cg12035144), and FAM8A1 (cg10154826). The remaining seven CpG sites did not annotate to genes (cg13835168, cg12384790, cg27061576, cg23073974, cg0749225, cg26781321, and cg06723525) (Fig. 6 and Table 4). To further examine the differential methylation results associated with smokers vs. non-smokers and COPD vs. non-smokers, pathway analyses were performed using IPA. The possible differentially methylated genes identified with unadjusted raw $P$ value $P<0.001$ revealed significant enrichment of genes related to top diseases and biological functions (Tables 5 and 6) including enriched canonical pathways (Table 7).

\section{Pyrosequencing analysis}

Among the significant differentially methylated genes/ probes identified in smokers vs. non-smokers and COPD vs. non-smokers based on their gene ontology (GO) analysis that directly relates to the biological functions, we chose a subset of genes for validation using pyrosequencing. In our pyrosequencing validation approach, the most significant subset of genes with CpG sites identified from our genome-wide DNA methylation analysis was selected based on their location (CpG island). It is evident that $\mathrm{CpG}$ island in a gene is associated with high CG density region (promoter or regulatory element), and the methylation status of which indirectly co-relates to its gene expression. Validating the differential methylation status of the specific subset of genes by pyrosequencing will further enhances the utility of those suggestive genes to be developed as a novel epigenetic signatures. Thus, DNA methylation can be used as a tool for the development of epigenetic-based diagnostic and therapeutic strategies in smokers and patients with COPD.

Table 4 Top 10 differentially methylated CpG sites in COPD compared to smokers, sorted by $P$ value

\begin{tabular}{|c|c|c|c|c|c|c|}
\hline CpG site & Gene symbol & Chromosome & aProbe Type & $P$ value & ${ }^{\mathrm{b}}$ Gene location of first annotated transcript & $\bar{c}$ Island location \\
\hline cg06723525 & & 10 & ॥ & 8.96E-05 & & S_Shore \\
\hline cg08236285 & CSMD1 & 8 & $\|$ & $6.95 \mathrm{E}-05$ & Body & \\
\hline cg09749225 & & 6 & । & $8.57 \mathrm{E}-05$ & & Island \\
\hline $\operatorname{cg} 10154826$ & FAM8A1 & 6 & $\|$ & 9.94E-05 & $1^{\text {st }}$ Exon & Island \\
\hline cg12035144 & LOC285830 & 6 & ॥ & $7.28 \mathrm{E}-05$ & TSS1500 & S_Shore \\
\hline cg12384790 & & 12 & ॥ & $1.96 \mathrm{E}-05$ & & N_Shelf \\
\hline cg13835168 & & 6 & ॥ & $1.64 \mathrm{E}-05$ & & \\
\hline cg23073974 & & 2 & ॥ & 5.27E-05 & & \\
\hline cg26781321 & & 8 & $\|$ & 8.79E-05 & & \\
\hline cg27061576 & & 15 & $\|$ & $2.28 \mathrm{E}-05$ & & \\
\hline
\end{tabular}

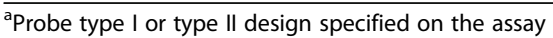

b Location relative to the first listed transcript

${ }^{c} N_{-}$and $S_{-}$denote the upstream and downstream end of the island region, respectively 

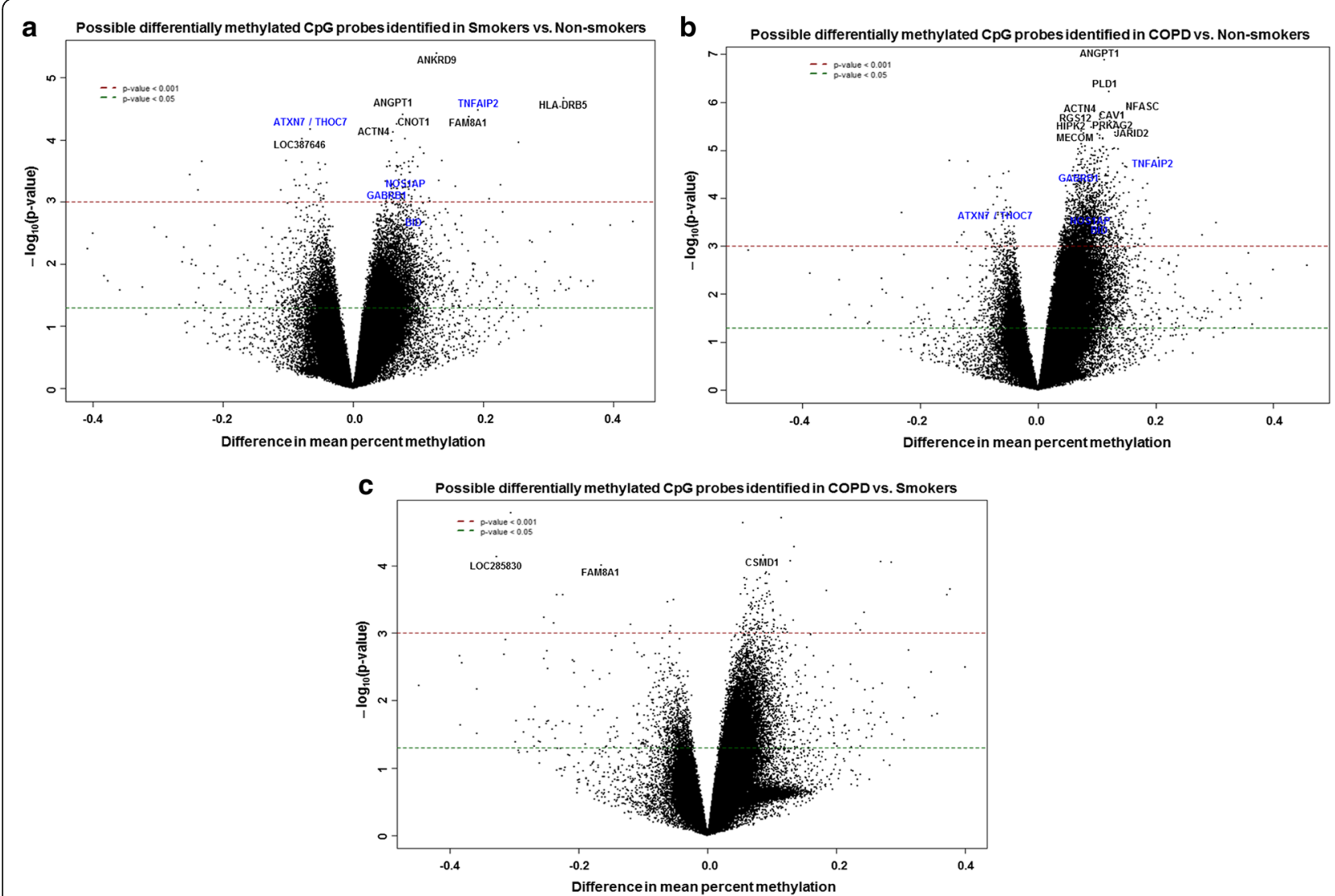

Fig. 2 Volcano plots showing possible differentially methylated CpG sites identified in this study. a Differential methylation analysis revealed top $10 \mathrm{CpG}$ sites and their genes significantly associated with smokers with $P<0.001$. Difference in mean percent methylation represents the difference in mean methylation between smokers vs. non-smokers (control). b Differential methylation analysis revealed top $10 \mathrm{CpG}$ sites and their genes significantly associated with COPD with $P<0.001$. Difference in mean percent methylation represents the difference in mean methylation between COPD vs. non-smokers (control). c Differential methylation analysis revealed CpG sites and their genes significantly associated with COPD compared to smokers with $P<0.001$. Difference in mean percent methylation represents the difference in mean methylation between COPD vs. Smokers (control). The $y$ axis represents the negative $\log P$ value of their association. The genes marked in blue color are the once validated by pyrosequencing analysis. The red and green dotted horizontal lines indicate the genome-wide significance threshold of $P<0.001$ and $P<0.05$, respectively. The top candidates in each pairwise comparison were selected by test statistics and unadjusted $P$ values for comparisons $(P<0.001$ is commonly used as a cutoff for relatively small unadjusted raw $P$ value)

In our genome-wide DNA methylation analysis, NOS1AP (cg26663636), TNFAIP2 (cg18620571), GABRB1 (cg15393297), and BID (cg01388022) are hypermethylated both in smoker and COPD compared to nonsmokers group (Fig. 7). We also selected ATXN7 and THOC7 genes (cg07753241) to validate since they both share the promoter (CpG island) that was hypomethylated in smoker and COPD in our genome-wide DNA methylation analysis (Fig. 8). Besides ATXN7 and THOC7 all the other genes (NOS1AP, TNFAIP2, GABRB1, and $B I D$ ) selected for pyrosequencing were significantly hyper-methylated in $\mathrm{CpG}$ island which is located in the gene body. Additionally, we included AHRR (cg21161138) [26] and SERPINA1 (cg02181506) [11] which were shown to be significantly hypo-methylated in smokers and patients with COPD based on prior studies as part of the pyrosequencing validation in this study. We designed pyrosequencing primer sets for the above mentioned subset of genes (Additional file 1: Figure S3-S9 and Table 8 ). Of the five different genes validated by pyrosequencing, NOS1AP was significantly hyper-methylated in smokers and COPD compared to non-smokers group (Fig. 8). Interestingly, we found that most of the CpG sites (out of the $11 \mathrm{CpG}$ sites) analyzed for NOS1AP showed significant hyper-methylation in smokers and COPD groups compared to non-smokers. Furthermore, when we combined the methylation percentage from all the $11 \mathrm{CpG}$ sites for NOS1AP, we found significant hyper-methylation in smokers and COPD compared to the non-smokers group (Additional file 1: Figure S10). Pyrosequencing validations for TNFAIP2 CpG site including additional $10 \mathrm{CpG}$ sites assayed were not significant both in smokers and COPD (Additional file 1: Figure S11). Validation for BID CpG site was not significant in smokers and COPD compared to 

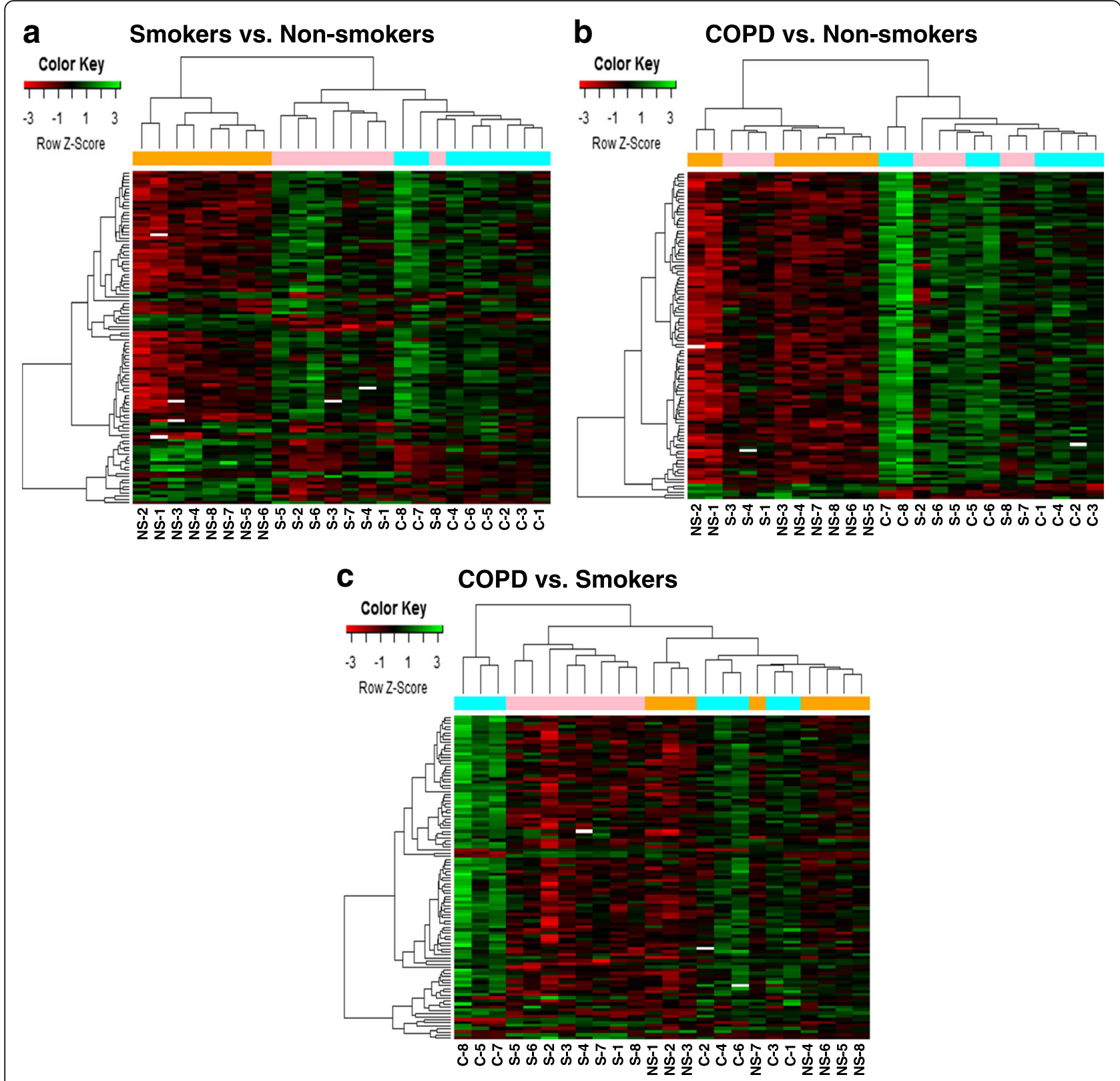

Fig. 3 Hierarchical cluster analysis of differentially methylated CpG sites identified in this study. a Heatmap of top 100 differentially methylated CpG sites in smokers vs. non-smokers group. $\mathbf{b}$ Heatmap of top 100 differentially methylated CpG sites in COPD vs. non-smokers group. $\mathbf{c}$ Heatmap of top 100 differentially methylated CpG sites in COPD vs. smokers group. Additionally for cluster analysis, we have also included the five genes that were chosen for validation for pyrosequencing along with the top 100 genes. The green color in the heatmap denotes hyper-methylated loci, and the red color in the heatmap denotes the hypo-methylated loci. Target id and gene list for the top 100 differentially methylated probes (a-c) are included in the Additional file 11: Table S10, Additional file 12: Table S11 and Additional file 13: Table S12

non-smokers groups. Instead, all the additional three CpG sites that were located close to the identified CpG site for BID showed significant hyper-methylation in smokers and COPD compared to the non-smokers group (Additional file 1: Figure S12). Our pyrosequencing analysis showed that the CpG site for GABRB1 was significantly hyper-methylated in smokers, although not significant in COPD compared to the non-smokers group. All the additional CpG sites validated for GABRB1 were not significant in smokers and COPD (Additional file 1: Figure S12). The CpG sites validated by pyrosequencing for ATXN7 and THOC7 for hypo-methylation in smokers and COPD compared to non-smokers group were not significant (Additional file 1: Figure S13). 


\section{cg14531093 (ANKRD9) cg15011943 (HLA-DRB5) cg22837763 (ANGPT1) cg10154826 (FAM8A1)}
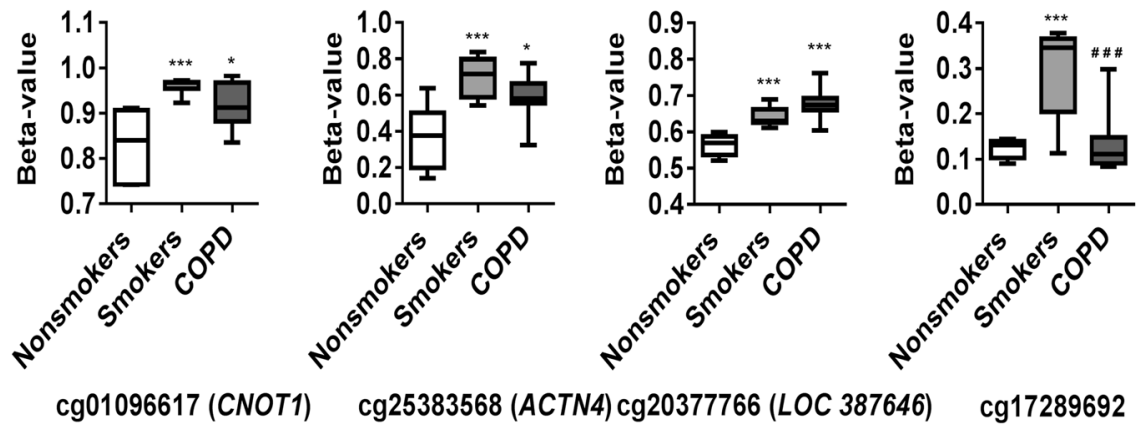

cg25383568 (ACTN4) cg20377766 (LOC 387646) cg17289692
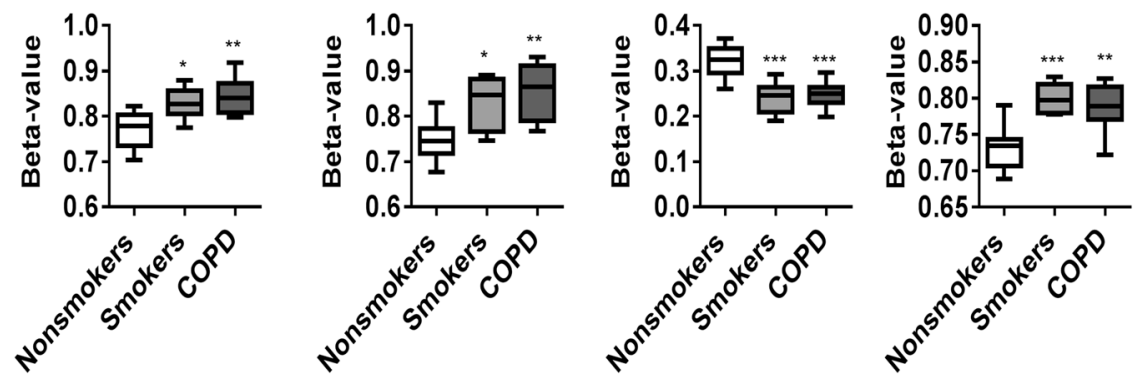

Fig. 4 Differentially methylated CpG sites associated with smokers. Differential methylation analysis revealed CpG sites in genes significantly associated with smokers with a $P$ value less than 0.05 . Difference in mean beta values represents the difference in mean methylation between smokers and non-smokers (controls). The $y$ axis represents the beta value. The gene symbol associated with CpG probes are provided in parenthesis. Data are represented as a box plot which displays the full range of variation (from min to max), the likely range of variation (the IQR) and a typical value (the median) for $n=8$ /group, and the significance determined using one-way ANOVA (Tukey's multiple comparisons test). ${ }^{*} P<0.05$, ${ }^{* *} P<0.01$, ${ }^{* * *} P<0.001$, vs. non-smokers; ${ }^{\# \#} P<0.001$ vs. smokers

\section{Discussion}

Epigenetic modifications may play a critical role in regulating expression of genes involved in molecular pathways and cellular processes relevant to the pathogenesis of COPD [1]. Recently, we have reported cigarette smoke differentially regulates gene expression profiles of epigenetic chromatin modification enzymes and DNA methyltransferases in vitro in $\mathrm{H} 292$ cell and in vivo in mouse lung [27]. Previous genome-wide DNA methylation studies were focused on DNA methylation associated with smoking and COPD using various sample types, such as whole blood, lymphoblasts, pulmonary macrophages, sputum, buccal, small airway cells, and lung tissues $[5-14,17,18]$. In the present study, we used lung parenchymal tissue DNA to perform genome-wide DNA methylation analysis using the Illumina's Infinium 450K methylation BeadChip and validated selective $\mathrm{CpG}$ sites from a subset of genes including NOS1AP, BID, and GABRB1 by pyrosequencing analysis. We have identified several novel target CpG sites of specific genes by genome-wide methylation analysis using lung tissue DNA from smokers and patients with COPD based on their biological functions.

Pathway analysis revealed possible genes differentially methylated in our genome-wide study among the smokers and COPD group that belong to cardiovascular disease and respiratory disease, respectively, under the disease and disorders categories. Based on their molecular and cellular functions, the identified top differentially methylated probes (DMPs) among smokers include cell morphology, cell-to-cell signaling and interaction, cellular assembly and organization, cell cycle, and cell death and survival. Additionally, DMPs among the patients with COPD showed cellular development, cell death and survival, cell morphology, cellular assembly and organization, and cellular function and maintenance as their top molecular and cellular functions. Further, our pyrosequencing data from NOS1AP, BID, and GABRB1 CpG sites analysis validates hyper-methylation in smokers and COPD compared to non-smokers. These genes are part of the relevant pathways in the pathogenesis of COPD, e.g., oxidative stress and mitochondrial dysfunction/autophagy. We speculate that altered DNA methylation status may affect gene expression of NOS1AP, BID, and GABRB1 genes in lung tissues of smokers and patients with COPD and perturb the key cellular pathways involved in cellular senescence/autophagy/apoptosis. Future studies will address this issue by validating this hypothesis using the same samples for both DNA methylation and gene expression analysis.

It is known that promoter hyper-methylation can cause gene silencing. Hence, the results of our genome wide 

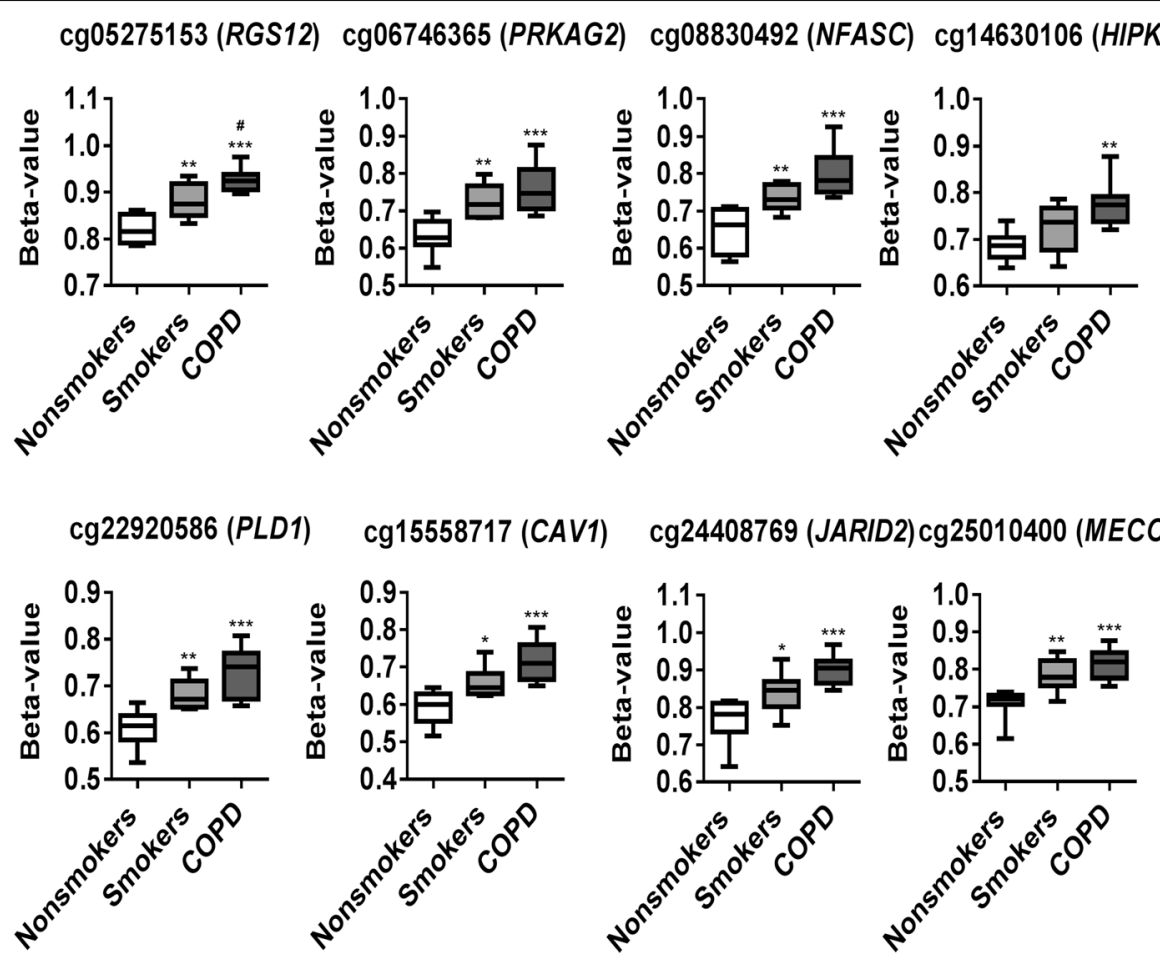

Fig. 5 Differentially methylated CpG sites associated with COPD. Differential methylation analysis revealed CpG sites in genes significantly associated with COPD with a $P$ value less than 0.05 . Difference in mean beta values represents the difference in mean methylation between COPD and non-smokers (controls). The $y$ axis represents the beta value. The gene symbol associated with $\mathrm{CpG}$ probes are provided in parenthesis. Data are represented as a box plot which displays the full range of variation (from min to max), the likely range of variation (the IQR) and a typical value (the median) for $n=8 /$ group, and the significance determined using one-way ANOVA (Tukey's multiple comparisons test). ${ }^{*} P<0.05$, ${ }^{* *} P<0.01,{ }^{* * *} P<0.001$, vs. non-smokers; ${ }^{\#} P<0.05$ vs. smokers

analysis may provide insights into the involvement of possible target genes in the pathogenesis of smoking-related chronic lung diseases by looking into which genes show changes in promoter DNA methylation. Among the top significant genes which are hyper-methylated in smokers include ANKRD9, HLA-DRB5 TNFAIP2, ANGPT1, FAM8A1, ATXN7, THOC7, CNOT1, and ACTN4. Similarly, when we compared COPD group with the nonsmokers, all the top differentially methylated CpG sites significantly hyper-methylated includes ANGPT1, PLD1, NFASC, ACTN4, RGS12, CAV1, PRKAG2, JARID2, HIPK2, and $M E C O M$. Some of the top differentially methylated genes, such as ANKRD9, CAV1, and JARID2, were previously known to be highly expressed in basal cells [28] significantly hyper-methylated in smokers and COPD groups confirmed by genome-wide DNA methylation analysis. A recent study suggests that caveolin-1 (Cav1) as a critical regulatory protein in pathological mechanisms of chronic inflammatory lung diseases. Loss of CAV1 contributes to an imbalance in the Th17/Treg cell in patients with COPD [29]. Additionally, two other genes TNFAIP2 and ANGPT1 are identified to be hyper-methylated as smoking-responsive genes discovered by microarray and RNA sequencing approaches [30, 31]. Recently,
CpG island hyper-methylation of $A N K R D 18 B$ was associated with decreased expression of $A N K R D 18 B$ in lung cancer tissues and cell lines compared to normal lung tissues [32]. Chi et al. reported that ANKRD11 was one among the other genes (ANKHD1 and LGALS2) with both regulatory DNA methylation sites in circulating monocytes and their mRNA expression that was associated with air pollution [33]. Another report showed HLA-DRB5 gene (major histocompatibility complex, class II, DR beta 5) among the expression quantitative trait loci (eQTLs) expressed in neutrophils that may be involved in immune-recognition/regulation and mitochondrial function [34]. Wan et al. has shown association of genes such as HIPK3(homeodomain-interacting protein kinase 3), a serine-threonine protein kinase that participates in cAMP-mediated steroidogenesis involved in the synthesis and regulation of steroid hormones, was differentially methylated in COPD who were under systemic steroid use [9]. Furthermore, these CpG sites and loci differentially methylated in smokers and COPD can be used as epigenetic signature in smokers and COPD based on their methylation status. Overall, several gene families identified (i.e., ANGPT, ANKRD, HIPK, and HLA-DRB) among the top differentially methylated $\mathrm{CpG}$ sites from 


\section{cg08236285 (CSMD1) cg12035144 (LOC285830) cg09749225 cg26781321}
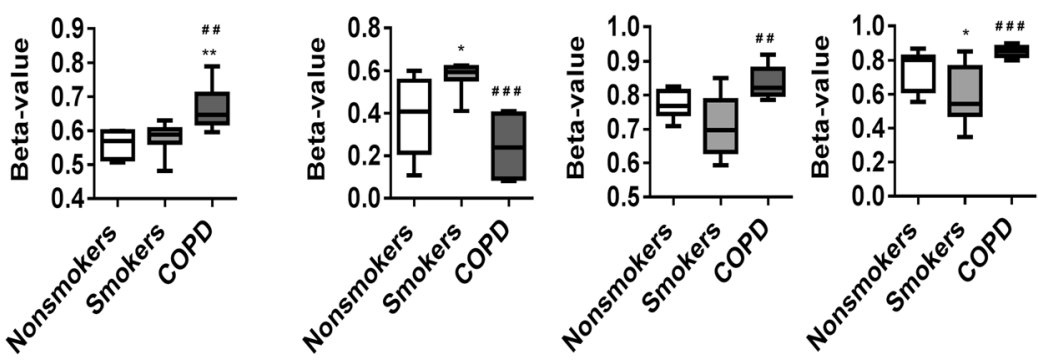

$\operatorname{cg} 06723525$
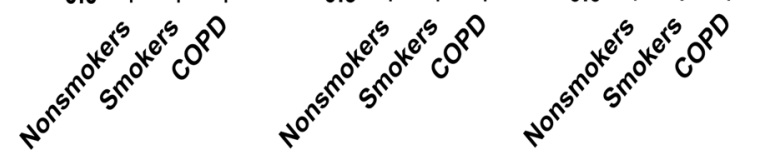

$\operatorname{cg} 13835168$

$\operatorname{cg} 12384790$

$\operatorname{cg} 27061576$

$\operatorname{cg} 23073974$

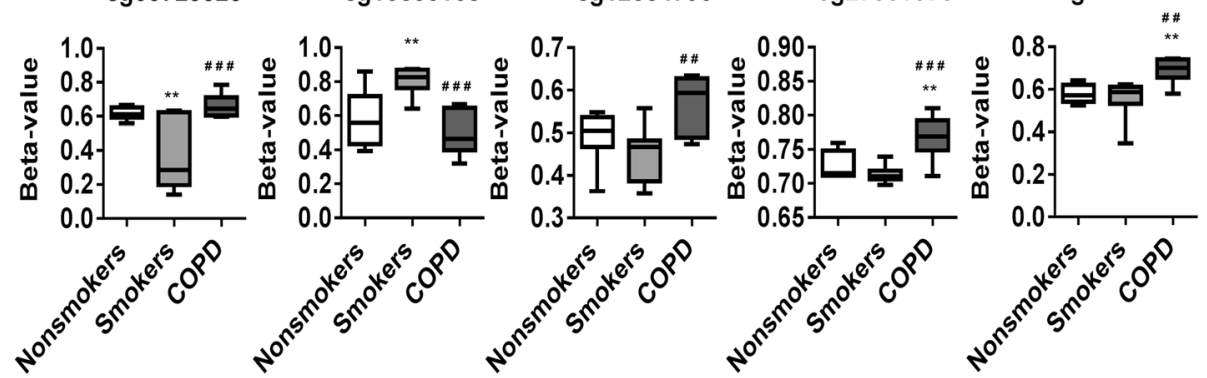

Fig. 6 Differentially methylated CpG sites associated with COPD vs. smokers. Differential methylation analysis revealed CpG sites in genes significantly associated with smokers vs. COPD with a $P$ value less than 0.05 . Difference in mean beta values represents the difference in mean methylation between smokers and COPD. The $y$ axis represents the beta value. The gene symbol associated with CpG probes are provided in parenthesis. Data are represented as a box plot which displays the full range of variation (from min to max), the likely range of variation (the IQR) and a typical value (the median) for $n=8 /$ group, and the significance determined using one-way ANOVA (Tukey's multiple comparisons test).

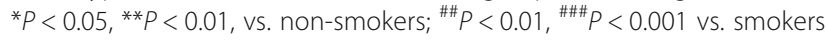

this study have been previously implicated as responsive genes in smokers and patients with COPD. This is based on their transcriptomics/DNA methylation data suggesting their role in the pathogenesis of smoking-related chronic lung diseases including COPD.

Our pyrosequencing data validates DNA methylation status of three candidate genes (NOS1AP, BID, and $G A B R B 1)$ out of five genes that was identified from the genome-wide DNA methylation analysis. Nitric oxide synthase 1 adaptor protein (NOS1AP) is a cytosolic protein that binds to the signaling molecule nNOS (neuronal nitric oxide synthase) that plays an important role in relieving hemodynamic force, and thereby aids in protecting the arterial walls from vascular inflammation [35]. It is known that cardiovascular disease and co-morbidities are associated with COPD [36], COPD patients have a threefold risk of developing ischemic heart disease and lung cancer compared to smokers without clinical manifestation of COPD. These findings support that vascular dysfunction and systemic inflammation are linked to COPD $[37,38]$. Interestingly, NOS1AP DNA methylation data from this study is in line with the previous report. Hypermethylation of NOS1AP promoter is shown to be associated with intracranial aneurysm and brain arteriovenous malformation as a result of regular tobacco use [39] NOS1AP has been implicated in several human neurodegenerative diseases, such as cardiovascular disorders (stroke), psychiatric disorders, and posttraumatic stress disorders [40]. Variation among oxidant stress pathway genes including NOS1AP in recipients and donors has been shown to be associated with primary graft dysfunction after lung transplantation [41]. Earlier studies show association of NOS1AP differential methylation in smokers without any validation [42-44]. To our knowledge, ours is the first study to identify NOS1AP hyper-methylation in lung parenchymal tissues by genome-wide DNA methylation and pyrosequencing analysis showing association of nNOS with smokers and patients with COPD who are at risk to cardiovascular diseases (co-morbidities).

Recently, we and others have shown role of mitochondrial dysfunction (defective mitophagy) in cigarette smoke-induced cellular senescence in lung cells (epithelial and fibroblasts) in vitro and mouse lungs in vivo as well as in smokers and patients with COPD [45, 46]. $B I D$ (BH3-interacting domain death agonist) protein is a member of the $B C L-2$ family of cell death regulators. $B I D$ functions in mitochondria for apoptosis during mitotic arrest [47] and engages a ROS-dependent, local inter-mitochondrial potentiation mechanism that amplifies the apoptotic signal [48]. BID CpG site was significantly hyper-methylated among the smokers and COPD 
Table 5 Top diseases and biological functions associated with differentially methylated genes between smokers vs. non-smokers

\begin{tabular}{lll}
\hline Diseases and disorders & $P$ value & No. of molecules \\
Hereditary disorder & $1.44 \mathrm{E}-02-8.85 \mathrm{E}-05$ & 3 \\
Organismal injury and abnormalities & $4.84 \mathrm{E}-02-8.85 \mathrm{E}-05$ & 8 \\
Renal and urological disease & $2.82 \mathrm{E}-02-8.85 \mathrm{E}-05$ & 2 \\
Cancer & $4.84 \mathrm{E}-02-3.80 \mathrm{E}-04$ & 8 \\
Cardiovascular disease & $3.45 \mathrm{E}-02-3.803 \mathrm{E}-04$ & 1 \\
Molecular and cellular functions & $P$ value & No. of molecules \\
Cell morphology & $3.48 \mathrm{E}-02-3.80 \mathrm{E}-04$ & 2 \\
Cell-to-cell signaling and interaction & $4.07 \mathrm{E}-02-3.80 \mathrm{E}-04$ & 2 \\
Cellular assembly and organization & $4.44 \mathrm{E}-02-3.80 \mathrm{E}-04$ & 3 \\
Cell cycle & $3.42 \mathrm{E}-03-7.61 \mathrm{E}-04$ & 2 \\
Cell death and survival & $4.45 \mathrm{E}-02-7.61 \mathrm{E}-04$ & 2 \\
Physiological system development and function & $P$ value & No. of molecules \\
Cardiovascular system development and function & $4.07 \mathrm{E}-02-3.80 \mathrm{E}-04$ & 3 \\
Embryonic development & $3.26 \mathrm{E}-02-3.80 \mathrm{E}-04$ & 4 \\
Lymphoid tissue structure and development & $6.07 \mathrm{E}-02-3.80 \mathrm{E}-04$ & 1 \\
Organismal development & $2.67 \mathrm{E}-02-3.80 \mathrm{E}-04$ & 3 \\
Organ morphology & $2.52 \mathrm{E}-02-3.80 \mathrm{E}-04$ & 3 \\
\hline
\end{tabular}

group in the $\mathrm{CpG}$ island based on our genome-wide DNA methylation analysis. Our DNA methylation for $B I D$ CpG site directly or indirectly affirms the involvement of apoptotic mechanism including mitochondrial dysfunction role in the pathogenesis of COPD $[49,50]$.

GABRB1 (gamma-aminobutyric acid type A receptor beta1 subunit) gene encodes for beta 1 subunit of the GABA A receptor, which is responsible for mediating inhibitory neurotransmission in the thalamus [51]. A previous report shows its role in alveolar fluid homeostasis of alveolar epithelial type II cells [52]. Gene expression changes caused by GABAergic system during smoking resulted in marked upregulation of GAD67 expression in both the large and small airway epithelium of healthy smokers compared to healthy non-smokers. This altered GAD67 expression was significantly

Table 6 Top diseases and biological functions associated with differentially methylated genes between COPD vs. non-smokers

\begin{tabular}{lll}
\hline Diseases and disorders & $P$ value & No. of molecules \\
Organismal injury and abnormalities & $7.91 \mathrm{E}-03-1.41 \mathrm{E}-04$ & 40 \\
Cancer & $7.91 \mathrm{E}-03-1.53 \mathrm{E}-04$ & 32 \\
Connective tissue disorders & $7.91 \mathrm{E}-03-3.69 \mathrm{E}-04$ & 8 \\
Dermatological diseases and conditions & $7.91 \mathrm{E}-03-5.12 \mathrm{E}-04$ & 13 \\
Respiratory disease & $7.91 \mathrm{E}-03-5.43 \mathrm{E}-04$ & 9 \\
Molecular and cellular functions & $P$ value & No. of molecules \\
Cellular development & $7.91 \mathrm{E}-03-3.40 \mathrm{E}-10$ & 58 \\
Cell death and survival & $7.91 \mathrm{E}-03-1.55 \mathrm{E}-07$ & 49 \\
Cell morphology & $7.91 \mathrm{E}-03-3.15 \mathrm{E}-07$ & 43 \\
Cellular assembly and organization & $7.91 \mathrm{E}-03-3.15 \mathrm{E}-07$ & 32 \\
Cellular function and maintenance & $7.91 \mathrm{E}-03-3.15 \mathrm{E}-07$ & 44 \\
Physiological system development and function & $P$ value & No. of molecules \\
Nervous system development and function & $7.91 \mathrm{E}-03-3.33 \mathrm{E}-09$ & 49 \\
Tissue morphology & $7.91 \mathrm{E}-03-2.60 \mathrm{E}-07$ & 49 \\
Tissue development & $7.91 \mathrm{E}-03-3.15 \mathrm{E}-07$ & 56 \\
Organ morphology & $7.91 \mathrm{E}-03-4.93 \mathrm{E}-07$ & 32 \\
Organismal development & $7.91 \mathrm{E}-03-4.93 \mathrm{E}-07$ & 60 \\
\hline
\end{tabular}


Table 7 Canonical pathways in ingenuity pathway analysis associated with differentially methylated genes between smokers vs. non-smokers and COPD vs. non-smokers

\begin{tabular}{lll}
\hline Smokers vs. non-smokers & & \\
Canonical pathways & $P$ value & Overlap \\
B cell development & $1.25 \mathrm{E}-02$ & $3.0 \% 1 / 33$ \\
Antigen presentation pathway & $1.40 \mathrm{E}-02$ & $2.7 \% 1 / 37$ \\
Autoimmune thyroid disease signaling & $1.77 \mathrm{E}-02$ & $2.1 \% 1 / 47$ \\
Graft-vs.-host disease signaling & $1.81 \mathrm{E}-02$ & $2.1 \% 1 / 48$ \\
Nur77 signaling in T lymphocytes & $2.15 \mathrm{E}-02$ & $1.8 \% 1 / 57$ \\
COPD vs. non-smokers & & \\
Canonical pathways & $P$ value & Overlap \\
G Beta gamma signaling & $5.35 \mathrm{E}-06$ & $8.1 \% 7 / 86$ \\
GNRH signaling & $6.42 \mathrm{E}-06$ & $6.5 \% 8 / 124$ \\
Molecular mechanisms of cancer & $6.98 \mathrm{E}-06$ & $3.6 \% 13 / 366$ \\
Role of NFAT in cardiac hypertrophy & $1.82 \mathrm{E}-05$ & $4.8 \% 9 / 187$ \\
nNOS signaling in neurons & $2.74 \mathrm{E}-05$ & $11.1 \% 5 / 45$ \\
\hline
\end{tabular}

correlated with increased $M U C 5 A C$ gene expression during smoking [53]. Our genome-wide DNA methylation data show significant hyper-methylation of GABRB1 in smokers and COPD. Nicotine can affect gene expression of DNA methyltransferase 1 (DNMT1) that directly or indirectly influences the promoter methylation status of GABAergic neurons, thereby providing a plausible link to nicotine addiction [54]. However, it remains unclear how GABRB1 functions as ligand-gated chloride channel during cigarette smokeinduced pulmonary toxic responses in the lungs of smokers and patients with COPD. Likewise, understanding the DNA methylation signature in lung tissues of smokers and COPD patients will enable us to discover the possibility of using selective epigeneticbased drugs that can attenuate progression of COPD in smokers.

In this study, we also included Serpin family A member 1 (SERPINA1) CpG site (cg02181506) and aryl hydrocarbon receptor repressor (AHRR) CpG site (cg21161138) for analysis along with the other CpG

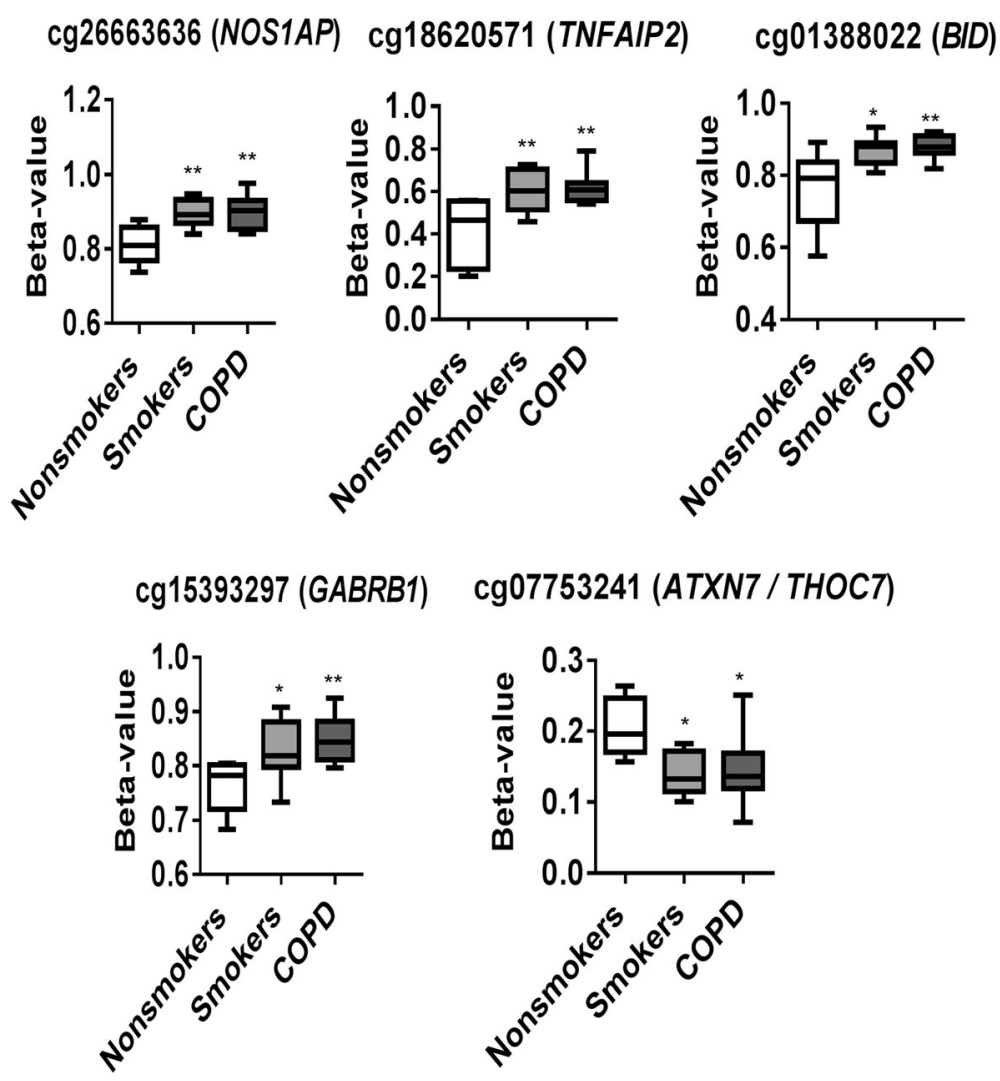

Fig. 7 Differentially methylated CpG sites identified from genome-wide methylation profiling for pyrosequencing validation. Differential methylation analysis revealed CpG sites in genes significantly associated with smokers and COPD compared to non-smokers with a $P$ value less than 0.05 . Difference in mean beta values represents the difference in mean methylation between smokers and COPD compared to non-smokers (controls). The $y$ axis represents the beta value. The gene symbol associated with $\mathrm{CpG}$ probes are provided in parenthesis. Data are represented as a box plot which displays the full range of variation (from min to max), the likely range of variation (the IQR) and a typical value (the median) for $n=8 /$ group, and the significance determined using one-way ANOVA (Tukey's multiple comparisons test). ${ }^{*} P<0.05,{ }^{* *} P<0.01$, vs. non-smokers 


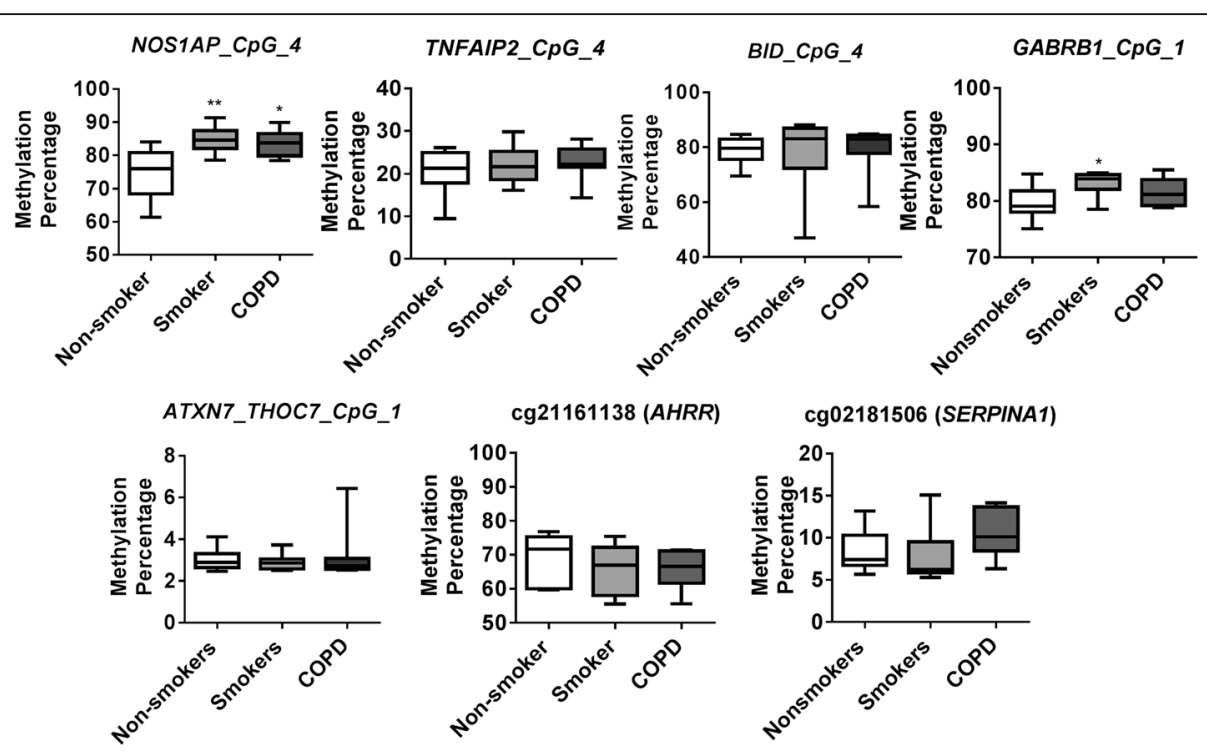

Fig. 8 Pyrosequencing validation of CPG sites identified in genome-wide DNA methylation analysis. NOS1AP locus cg2663636, TNFAIP2 locus cg18620571, BID locus cg01388022, GABRB1 locus cg15393297, and ATXN7 and THOC7 locus cg07753241 were validated along with AHRR locus cg21161138 and SERPINA1 locus cg02181506. Boxplots represent pyrosequencing methylation percentages between smokers and COPD compared to non-smokers control. Data are represented as box plot which displays the full range of variation (from min to max), the likely range of variation (the IQR) and a typical value (the median) for $n=8 /$ group, and the significance determined using one-way ANOVA (Tukey's multiple comparisons test). ${ }^{*} P<0.05,{ }^{* *} P<0.01$, vs. non-smokers

sites for validation even though they were not identified in our genome-wide methylation analysis. Surprisingly, our pyrosequencing data for SERPINA1 and AHRR hypo-methylation in smokers and patients with COPD did not show significant changes. SERPINA1 encodes for alpha-1-antitrypsin deficiency that contributes to the genetic susceptibility of COPD. Previously, SERPINA1 hypo-methylation using whole blood DNA has been shown in patients with COPD correlated with lower lung function [11]. In this study, pyrosequencing analysis for SERPINA1 showed correlation in the methylation status (methylation percentages) among the non-smoker groups. However, we were unable to see any significant hypomethylation of SERPINA1 CpG site in smokers and COPD groups compared to non-smokers. Similarly, smokinginduced DNA methylation alteration in AHRR has been evidently documented using DNA from lymphoblasts, alveolar macrophages, WBCs, and whole blood, suggesting

Table $8 \mathrm{CpG}$ pyrosequencing primer sequences

\begin{tabular}{|c|c|c|}
\hline Gene & Forward primer $5^{\prime}$ to $3^{\prime}$ & Reverse primer 3' to $5^{\prime}$ \\
\hline \multirow[t]{2}{*}{ NOS1AP (cg26663636) } & TGTTTGGTGAAGTTGGAGTGT & \multirow[t]{2}{*}{${ }^{\mathrm{a} C C C A A T T C C T A C C T C T A C A A A A C A T T C A ~}$} \\
\hline & Sequencing primer: GGTTTGGAGTTATTAAGTT & \\
\hline \multirow[t]{2}{*}{ TNFAIP2 (cg18620571) } & GTTAGAGTAAGGTGGAGG & \multirow[t]{2}{*}{${ }^{\mathrm{a} C C A A A T C C T C C T T C A T A A T A C ~}$} \\
\hline & Sequencing primer: AGAGTAAGGTGGAGG & \\
\hline \multirow[t]{2}{*}{ BID (cg01388022) } & GAGTTAAATAATGGGTGTTTATGGAT & \multirow[t]{2}{*}{ 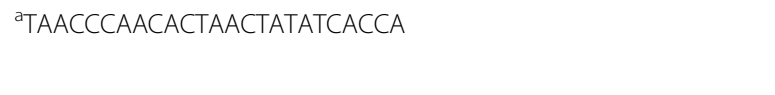 } \\
\hline & Sequencing primer: ATGGAGAGAGTAGGT & \\
\hline \multirow[t]{2}{*}{ GABRB1 (cg15393297) } & ${ }^{\text {aGTTAATGAAAATAGGTAAAGGTITTGTAAT }}$ & AACCACTTATCTATAAAATTCACATC \\
\hline & \multicolumn{2}{|c|}{ Sequencing primer: CTATCATAAAAATACATAATAACC } \\
\hline \multirow[t]{2}{*}{ ATXN7/THOC7 (cg07753241) } & ${ }^{\mathrm{a} T A G G G G T T T A G G G G A G G T T A G}$ & СТССТССТССАТАAСТАТТТАСС \\
\hline & \multicolumn{2}{|l|}{ Sequencing primer: ACTACATTATAAAAATTTAACCT } \\
\hline \multirow[t]{2}{*}{ AHRR (cg21161138) } & GGTTGGTGGTGTAGGATATATT & \multirow[t]{2}{*}{${ }^{\mathrm{a} G A C G G G A C A C C G C T G A T C G T T T A A C C C A T C C T A C C C A A A T C C T A A T A A T ~}$} \\
\hline & Sequencing primer: GGTTTAGGTTTAGGGA & \\
\hline \multirow[t]{2}{*}{ SERPINA1 (cg02181506) } & TITGGTTAGTTAGGATITGAGG & \multirow[t]{2}{*}{${ }^{a}$ AAACACTATACCCAAAACATACACTAC } \\
\hline & Sequencing primer: GGATTTTAGGGTTGTT & \\
\hline
\end{tabular}


the role of epigenetic effects mediated by CS on carcinogenesis and other related co-morbidities in a tissueindependent manner $[6,26,55-58]$. These studies have implicated the role of CS-mediated oxidative stress and inflammatory response associated with DNA methylation (hypo-methylation) in SERPINA1 and AHRR CpG sites. Our observations regarding the lack of correlation observed in SERPINA1 and AHRR hypo-methylation in smokers and COPD could be due to limited sample size, tissue heterogeneity (cell-type present in lung parenchymal tissue), and genetic variants present in our samples. We were unable to validate DNA methylation changes of the TNFAIP2 and ATXN7/THOC genes using pyrosequencing. The exact reason for lack on reproducibility of findings is not known. This inconsistency shows that validations are important to confirm the array-based DNA methylation analysis. Therefore, it is important to validate the identified CpG sites using another technique which is very specific for the target CpG sites, such as the pyrosequencing assays as used in this study.

In this study, we also identified DMR of oxidative stress-related genes, steroid-responsive genes, and cellular signaling genes linked to molecular and cellular functions (cell death and survival pathways). Previous studies have provided evidence that cigarette smoking plays an important role in epigenetic regulation of oxidative stress genes (e.g., glutamate-cysteine ligase catalytic subunit (GCLC)) and immune-related genes (IL-12RB2 and WIF-1) involved in the pathogenesis of COPD and lung cancer $(C C D C 37$, and $M A P 1 B)[5,11,59-61]$. Earlier studies have shown site-specific DNA methylation during steroid resistance in COPD, in smokers even after cessation (e.g., Factor II receptor-like 3 (F2RL3) and G-protein-coupled receptor 15 (GPR15)), and in newborns affected by maternal smoking (in utero, or early life exposure) leading to complications later in life $[9,10,16]$. Similarly, promoter methylation of p16 (CDKN2A) and GATA4 in sputum DNA from smokers showed significant correlation with decline in lung function [7]. Based on our observations and prior studies, we suggest DNA methylation does play an important role in regulation of oxidative stress, proinflammatory responses, and systemic steroid exposure in COPD $[5,9,12]$.

Our study has some limitations, such as limited number of human lung tissues used and cellular heterogeneity/ specific lung cell types which are affected by chronic cigarette smoking including the statistical analysis used for genome-wide DNA methylation analysis. However, this study is unique as it deals with parenchymal lung tissues which are involved in the pathogenesis of COPD. Further, our global DNA methylation data are indeed validated by pyrosequencing for the identified target genes. Future studies will additionally validate the identified genes using a larger cohort so as to determine the utility of suggestive genes as novel epigenetic signatures in smokers and patients with COPD.

\section{Conclusions}

In conclusion, we found novel genes/pathways associated with possible differential DNA methylation in smokers and patients with COPD using the Infinium HumanMethylation450 BeadChip. Based on genome-wide DNA methylation and functional network analysis, selective CpG sites (NOS1AP, BID, and GABRB1) differentially methylated in smokers and COPD compared to nonsmokers were validated by pyrosequencing. However, future studies will further validate differential methylation of target genes and their gene expression signatures in lung tissues from non-smokers, smokers, and patients with COPD. Overall, some of the top differentially methylated probes/genes identified in this study may be used as lung tissue-specific epigenetic signatures to stratify smoking exposure and predict the risk of developing smokingrelated chronic lung diseases including COPD, COPDexacerbations, and lung cancer after further validation of the suggested genes in a larger cohort. Further, a comprehensive, systematic assembly of COPD-related gene deregulation is required to integrate existing epigenome-wide DNA methylome and transcriptome signatures as they relate to COPD phenotypes and ultimately merge these data across platforms into a systems biology paradigm in COPD pathogenesis and its exacerbations.

\section{Additional files}

Additional file 1: Figures S1-S13. Figure S1.Boxplot shows distribution of pre-processed DNA methylation level ( $\beta$ values) from all the samples and different groups used for comparison. Figure S2.Venn diagrams shows DNA methylation loci that were identified to be overlapping and or shared between Non-smokers vs. Smokers compared to Non-smokers vs. COPD and Smoker vs. COPD. Figure S3-S9. Target sequence for the NOS1AP (cg26663636), TNFAIP2 (cg18620571), BID (cg01388022), GABRB1 (cg15393297), ATXN7/THOC7 (cg07753241), AHRR (cg21161138) and SERPINA1 (cg02181506) amplicons. Figure S10-13. Pyrosequencing validation of additional CpG sites for NOSTAP, TNFAIP2, BID, and ATXN7/THOC7. (PPTX $1.93 \mathrm{mb}$ )

Additional file 2: Table S1. CpGs differentially methylated in lung DNA in relation to smokers vs. non-smokers $(P<0.001)$. (CSV $1 \mathrm{~kb})$

Additional file 3: Table S2. CpGs differentially methylated in lung DNA in relation to COPD vs. non-smokers $(P<0.001)$. (CSV $37 \mathrm{~kb})$

Additional file 4: Table S3. CpGs differentially methylated in lung DNA in relation to COPD vs. smokers $(P<0.001)$. (CSV $1 \mathrm{~kb})$

Additional file 5: Table S4. CpGs differentially methylated in lung DNA in relation to smokers vs. non-smokers $(P<0.01)$. (CSV $16 \mathrm{~kb}$ )

Additional file 6: Table S5. CpGs differentially methylated in lung DNA in relation to COPD vs. non-smokers $(P<0.01)$. (CSV $273 \mathrm{~kb})$

Additional file 7: Table S6. CpGs differentially methylated in lung DNA in relation to COPD vs. smokers $(P<0.01)$. (CSV $19 \mathrm{~kb})$ 
Additional file 8: Table S7. CpGs differentially methylated in lung DNA in relation to smokers vs. non-smokers $(P<0.05)$. (CSV $1487 \mathrm{~kb})$

Additional file 9: Table S8. CpGs differentially methylated in lung DNA in relation to COPD vs. non-smokers $(P<0.05)$. (CSV $4866 \mathrm{~kb})$

Additional file 10: Table S9. CpGs differentially methylated in lung DNA in relation to COPD vs. smokers $(P<0.05)$. (CSV $1597 \mathrm{~kb}$ )

Additional file 11: Table S10. Top $100 \mathrm{CpGs}$ differentially methylated along with five $\mathrm{CpG}$ sites chosen for validation in lung DNA in relation to smokers vs. non-smokers shown in heatmap (Fig. 3a). (CSV 2 kb)

Additional file 12: Table S11. Top 100 CpGs differentially methylated along with five $\mathrm{CpG}$ sites chosen for validation in lung DNA in relation to COPD vs. non-smokers shown in heatmap (Fig. 3b). (CSV 2 kb)

Additional file 13: Table S12. Top 100 CpGs differentially methylated along with five $\mathrm{CpG}$ sites chosen for validation in lung DNA in relation to COPD vs. smokers shown in heatmap (Fig. 3c). (CSV 3 kb)

\section{Abbreviations}

ACTN4: Actinin alpha 4; AHRR: Aryl hydrocarbon receptor repressor AML: Acute myeloid leukemia; ANGPT1: Angiopoietin 1; ANKHD1: Ankyrin repeat and KH domain containing 1; ANKRD9: Ankyrin repeat domain 9; ATXN7: Ataxin 7; BID: BH3 interacting domain death agonist; CAV1: Caveolin 1; CCDC37: Coiled-coil domain containing 37; CNOT1: CCR4-NOT transcription complex subunit 1; COPD: Chronic obstructive pulmonary disease; CpG: Cytosine-phosphate-guanine; CS: Cigarette smoke; CSMD1: CUB and sushi multiple domains 1; DMBA: 7, 12-Dimethylbenz[a]anthracene; DMP: Differentially methylated probe; DNMT1: DNA methyltransferase 1; F2RL3: F2R-like thrombin/trypsin receptor 3; FAM8A1: Family with sequence similarity 8 member A1; FEV1: Forced expiratory volume in $1 \mathrm{~s}$; GABRB1: Gamma-aminobutyric acid type A receptor beta1 subunit; GAD67: Glutamate decarboxylase 67; GCLC: Glutamate-cysteine ligase catalytic subunit; GO: Gene ontology; GPR15: G protein-coupled receptor 15; HIPK2: Homeodomain interacting protein kinase 2; HIPK3: Homeodomain interacting protein kinase 3; HLA-DRB5: Major histocompatibility complex, class II, DR beta 5; IL12RB2: Interleukin 12 receptor subunit beta 2; IMA: Illumina methylation analyzer; IPA: Ingenuity pathway analysis; JARID2: Jumonji and AT-rich interaction domain containing 2; LGALS2: Galectin 2; MAP1B: Microtubule associated protein 1B; MECOM: MDS1 and EVI1 complex locus; MUC5AC: Mucin 5AC, oligomeric mucus/gel-forming; NFASC: Neurofascin; NOS1AP: Nitric oxide synthase 1 adaptor protein; PLD1: Phospholipase D1; PRKAG2: Protein kinase AMP-activated non-catalytic subunit gamma 2; PRKCA: Protein kinase $\mathrm{C}$ alpha; PRKCABP: Protein kinase C, alpha binding protein; RGS12: Regulator of G-protein signaling 12; ROS: Reactive oxygen species; SERPINA1: Serpin family A member 1; SNP: Singlenucleotide polymorphism; THOC7: THO complex 7; TNFAIP2: TNF alpha induced protein 2; WBC: White blood cells; WIF1: WNT inhibitory factor 1

\section{Acknowledgements}

Thanks to Drs. Jeffery Conroy (Genomics Shared Resource) and Dr. Song Liu (Bioinformatics) from Roswell Park Cancer Institute, Buffalo, NY for DNA methylation. DNA methylation analysis was performed by the Genomics Shared Resource supported by Roswell Park Cancer Institute and National Cancer Institute (NCI) grant P30CA016056. We thank the late Dr. Vuokko L Kinnula and Dr. Witold Mazur (University of Helsinki, Finland) for providing the human lung tissue samples from non-smokers, smokers, and patients with COPD for conducting this genome-wide DNA methylation analysis.

\section{Funding}

This study was supported by the NIH2R01HL085613 and 3R01HL085613-07S1 (to I.R.), American Lung Association RG-305393 (to I.K.S), and NIEHS Environmental Health Science Center grant P30-ES001247.

\section{Availability of data and materials}

The raw datasets supporting this study will be available in the NCBI Gene Expression Omnibus repository, GEO Series accession number: GSE92511.

\section{Authors' contributions}

IKS, QY, MS, and IR conceived and designed the experiments. IKS and QY performed the experiments. IKS and QY analyzed the data. BSB, MS, and DL contributed reagents for pyrosequencing analysis and statistical analysis. IKS and IR wrote the manuscript. All authors read and approved the final manuscript.

\section{Competing interests}

The authors declare that they have no competing interests.

\section{Consent for publication}

Not applicable.

\section{Ethics approval and consent to participate}

Lung tissue specimens were retrieved from patients of the Helsinki University Hospital $(H \cup H)$ in collaboration with the late Dr. Vuokko L Kinnula and Dr. Witold Mazur (University of Helsinki, Finland). The study was approved by the ethical committee of $\mathrm{HUH}$, and all the participants provided written informed consent. Samples from non-smokers, smokers, and patients with COPD based on the value of forced expiratory volume in $1 \mathrm{~s}\left(\mathrm{FEV}_{1}\right)$ were obtained from the tumor-free peripheral lung in operations for local tumors.

\section{Publisher's Note}

Springer Nature remains neutral with regard to jurisdictional claims in published maps and institutional affiliations.

\section{Author details}

${ }^{1}$ Department of Environmental Medicine, University of Rochester Medical Center, Box 850, 601 Elmwood Avenue, Rochester 14642, NY, USA. ${ }^{2}$ Department of Clinical \& Translational Research, University of Rochester Medical Center, Rochester, NY, USA. ${ }^{3}$ Department of Biostatistics and Bioinformatics, Roswell Park Cancer Institute, Buffalo, NY, USA. ${ }^{4}$ Heart and Lung Center, University of Helsinki and Helsinki University Hospital, Helsinki, Finland.

Received: 19 December 2016 Accepted: 29 March 2017

Published online: 14 April 2017

\section{References}

1. Yao H, Rahman I. Current concepts on oxidative/carbonyl stress, inflammation and epigenetics in pathogenesis of chronic obstructive pulmonary disease. Toxicol Appl Pharmacol. 2011;254:72-85.

2. Sundar IK, Mullapudi N, Yao H, Spivack SD, Rahman I. Lung cancer and its association with chronic obstructive pulmonary disease: update on nexus of epigenetics. Curr Opin Pulm Med. 2011;17:279-85.

3. Sundar IK, Yao H, Rahman I. Oxidative stress and chromatin remodeling in chronic obstructive pulmonary disease and smoking-related diseases. Antioxid Redox Signal. 2013;18:1956-71.

4. Morrow JD, Cho MH, Hersh CP, Pinto-Plata V, Celli B, Marchetti N, Criner G, Bueno R, Washko G, Glass K, et al. DNA methylation profiling in human lung tissue identifies genes associated with COPD. Epigenetics. 2016;11:730-39.

5. Cheng L, Liu J, Li B, Liu S, Li X, Tu H. Cigarette smoke-induced hypermethylation of the GCLC gene is associated with COPD. Chest. 2016;149:474-82.

6. Monick MM, Beach SR, Plume J, Sears R, Gerrard M, Brody GH, Philibert RA. Coordinated changes in AHRR methylation in lymphoblasts and pulmonary macrophages from smokers. Am J Med Genet B Neuropsychiatr Genet. 2012;159B:141-51.

7. Sood A, Petersen H, Blanchette CM, Meek P, Picchi MA, Belinsky SA, Tesfaigzi Y. Wood smoke exposure and gene promoter methylation are associated with increased risk for COPD in smokers. Am J Respir Crit Care Med. 2010;182:1098-104

8. Wan ES, Qiu W, Carey VJ, Morrow J, Bacherman H, Foreman MG, Hokanson JE, Bowler RP, Crapo JD, DeMeo DL. Smoking-associated site-specific differential methylation in buccal mucosa in the COPDGene Study. Am J Respir Cell Mol Biol. 2015;53:246-54.

9. Wan ES, Qiu W, Baccarelli A, Carey VJ, Bacherman H, Rennard SI, Agusti A, Anderson WH, Lomas DA, DeMeo DL. Systemic steroid exposure is associated with differential methylation in chronic obstructive pulmonary disease. Am J Respir Crit Care Med. 2012;186:1248-55.

10. Wan ES, Qiu W, Baccarelli A, Carey VJ, Bacherman H, Rennard SI, Agusti A, Anderson W, Lomas DA, Demeo DL. Cigarette smoking behaviors and time since quitting are associated with differential DNA methylation across the human genome. Hum Mol Genet. 2012;21:3073-82.

11. Qiu W, Baccarelli A, Carey VJ, Boutaoui N, Bacherman H, Klanderman B, Rennard S, Agusti A, Anderson W, Lomas DA, DeMeo DL. Variable DNA 
methylation is associated with chronic obstructive pulmonary disease and lung function. Am J Respir Crit Care Med. 2012;185:373-81.

12. Vucic EA, Chari R, Thu KL, Wilson IM, Cotton AM, Kennett JY, Zhang M, Lonergan KM, Steiling K, Brown CJ, et al. DNA methylation is globally disrupted and associated with expression changes in chronic obstructive pulmonary disease small airways. Am J Respir Cell Mol Biol. 2014;50:912-22.

13. Yoo S, Takikawa S, Geraghty P, Argmann C, Campbell J, Lin L, Huang T, Tu Z, Foronjy RF, Spira A, et al. Integrative analysis of DNA methylation and gene expression data identifies EPAS1 as a key regulator of COPD. PLoS Genet. 2015;11:e1004898.

14. Qiu W, Wan E, Morrow J, Cho MH, Crapo JD, Silverman EK, DeMeo DL. The impact of genetic variation and cigarette smoke on DNA methylation in current and former smokers from the COPDGene study. Epigenetics. 2015;10:1064-73.

15. Breitling LP, Yang R, Korn B, Burwinkel B, Brenner H. Tobacco-smokingrelated differential DNA methylation: $27 \mathrm{~K}$ discovery and replication. Am J Hum Genet. 2011;88:450-7.

16. Joubert BR, Haberg SE, Nilsen RM, Wang X, Vollset SE, Murphy SK, Huang Z, Hoyo C, Midttun O, Cupul-Uicab LA, et al. 450 K epigenome-wide scan identifies differential DNA methylation in newborns related to maternal smoking during pregnancy. Environ Health Perspect. 2012;120:1425-31.

17. Guzman L, Depix MS, Salinas AM, Roldan R, Aguayo F, Silva A, Vinet R. Analysis of aberrant methylation on promoter sequences of tumor suppressor genes and total DNA in sputum samples: a promising tool for early detection of COPD and lung cancer in smokers. Diagn Pathol. 2012;7:87.

18. Gao X, Zhang Y, Breitling LP, Brenner H: Relationship of tobacco smoking and smoking-related DNA methylation with epigenetic age acceleration. Oncotarget. 2016;7:46878-46889.

19. Busch R, Qiu W, Lasky-Su J, Morrow J, Criner G, DeMeo D. Differential DNA methylation marks and gene comethylation of COPD in African-Americans with COPD exacerbations. Respir Res. 2016;17:143.

20. Sundar IK, Yin Q, Baier BS, Yan L, Li D, Susiarjo M, Rahman I. DNA methylation profile in lungs of smokers and patients with COPD is associated with specific genes and cellular pathways, 1st BNMC Translational Genomics and Epigenomics Symposium, September 23-24th 2016 at Roswell Park Cancer Institute, Buffalo, NY. 2016.

21. Rajendrasozhan S, Yang SR, Kinnula VL, Rahman I. SIRT1, an antiinflammatory and antiaging protein, is decreased in lungs of patients with chronic obstructive pulmonary disease. Am J Respir Crit Care Med. 2008;177:861-70.

22. Yao H, Chung S, Hwang JW, Rajendrasozhan S, Sundar IK, Dean DA, McBurney MW, Guarente L, Gu W, Ronty M, et al. SIRT1 protects against emphysema via FOXO3-mediated reduction of premature senescence in mice. J Clin Invest. 2012;122:2032-45.

23. Aryee MJ, Jaffe AE, Corrada-Bravo H, Ladd-Acosta C, Feinberg AP, Hansen KD, Irizarry RA. Minfi: a flexible and comprehensive bioconductor package for the analysis of Infinium DNA methylation microarrays. Bioinformatics. 2014;30:1363-9.

24. Maksimovic J, Gordon L, Oshlack A. SWAN: subset-quantile within array normalization for illumina infinium HumanMethylation450 BeadChips. Genome Biol. 2012;13:R44.

25. Ritchie ME, Phipson B, Wu D, Hu Y, Law CW, Shi W, Smyth GK. Limma powers differential expression analyses for RNA-sequencing and microarray studies. Nucleic Acids Res. 2015;43:e47.

26. Shenker NS, Ueland PM, Polidoro S, van Veldhoven K, Ricceri F, Brown R, Flanagan JM, Vineis P. DNA methylation as a long-term biomarker of exposure to tobacco smoke. Epidemiology. 2013;24:712-6.

27. Sundar IK, Rahman I. Gene expression profiling of epigenetic chromatin modification enzymes and histone marks by cigarette smoke: Implications for COPD and lung cancer. Am J Physiol Lung Cell Mol Physiol. 2016;311: L1245-L1258.

28. Hackett NR, Shaykhiev R, Walters MS, Wang R, Zwick RK, Ferris B, Witover B, Salit J, Crystal RG. The human airway epithelial basal cell transcriptome. PLoS One. 2011;6:e18378

29. Sun N, Wei X, Wang J, Cheng Z, Sun W. Caveolin-1 promotes the imbalance of Th17/Treg in patients with chronic obstructive pulmonary disease. Inflammation. 2016;39:2008-15.

30. Dvorak A, Tilley AE, Shaykhiev R, Wang R, Crystal RG. Do airway epithelium air-liquid cultures represent the in vivo airway epithelium transcriptome? Am J Respir Cell Mol Biol. 2011:44:465-73.

31. Hackett NR, Butler MW, Shaykhiev R, Salit J, Omberg L, Rodriguez-Flores JL Mezey JG, Strulovici-Barel Y, Wang G, Didon L, Crystal RG. RNA-Seq quantification of the human small airway epithelium transcriptome. BMC Genomics. 2012;13:82.

32. Liu WB, Han F, Jiang $X$, Yin L, Chen HQ, Li YH, Liu Y, Cao J, Liu JY. Epigenetic regulation of ANKRD18B in lung cancer. Mol Carcinog. 2015;54:312-21.

33. Chi GC, Liu Y, MacDonald JW, Barr RG, Donohue KM, Hensley MD, Hou L, McCall CE, Reynolds LM, Siscovick DS, Kaufman JD. Long-term outdoor air pollution and DNA methylation in circulating monocytes: results from the Multi-Ethnic Study of Atherosclerosis (MESA). Environ Health. 2016;15:119.

34. Andiappan AK, Melchiotti R, Poh TY, Nah M, Puan KJ, Vigano E, Haase D, Yusof N, San Luis B, Lum J, et al. Genome-wide analysis of the genetic regulation of gene expression in human neutrophils. Nat Commun. 2015;6:7971.

35. Forstermann U, Sessa WC. Nitric oxide synthases: regulation and function. Eur Heart J. 2012;33:829-37. 837a-837d.

36. Crapo JD. Preclinical vascular disease identifies smokers at risk for COPD. Proc Natl Acad Sci U S A. 2010;107:8503-4.

37. Maclay JD, McAllister DA, Mills NL, Paterson FP, Ludlam CA, Drost EM, Newby DE, Macnee W. Vascular dysfunction in chronic obstructive pulmonary disease. Am J Respir Crit Care Med. 2009:180:513-20.

38. Sabit R, Bolton CE, Edwards PH, Pettit RJ, Evans WD, McEniery CM, Wilkinson IB, Cockcroft JR, Shale DJ. Arterial stiffness and osteoporosis in chronic obstructive pulmonary disease. Am J Respir Crit Care Med. 2007;175:1259-65.

39. Wang Z, Zhao J, Sun J, Nie S, Li K, Gao F, Zhang T, Duan S, Di Y, Huang Y, Gao X. Sex-dichotomous effects of NOS1AP promoter DNA methylation on intracranial aneurysm and brain arteriovenous malformation. Neurosci Lett. 2016;621:47-53.

40. Li LL, Melero-Fernandez de Mera RM, Chen J, Ba W, Kasri NN, Zhang M, Courtney MJ. Unexpected heterodivalent recruitment of NOS1AP to nNOS reveals multiple sites for pharmacological intervention in neuronal disease models. J Neurosci. 2015;35:7349-64.

41. Cantu E, Shah RJ, Lin W, Daye ZJ, Diamond JM, Suzuki Y, Ellis JH, Borders CF, Andah GA, Beduhn B, et al. Oxidant stress regulatory genetic variation in recipients and donors contributes to risk of primary graft dysfunction after lung transplantation. J Thorac Cardiovasc Surg. 2015;149:596-602.

42. Guida F, Sandanger TM, Castagne R, Campanella G, Polidoro S, Palli D, Krogh V, Tumino R, Sacerdote C, Panico S, et al. Dynamics of smokinginduced genome-wide methylation changes with time since smoking cessation. Hum Mol Genet. 2015;24:2349-59.

43. Besingi W, Johansson A. Smoke-related DNA methylation changes in the etiology of human disease. Hum Mol Genet. 2014;23:2290-7.

44. Zeilinger S, Kuhnel B, Klopp N, Baurecht H, Kleinschmidt A, Gieger C, Weidinger S, Lattka E, Adamski J, Peters A, et al. Tobacco smoking leads to extensive genome-wide changes in DNA methylation. PLoS One. 2013;8:e63812.

45. Ahmad T, Sundar IK, Lerner CA, Gerloff J, Tormos AM, Yao H, Rahman I. Impaired mitophagy leads to cigarette smoke stress-induced cellular senescence: implications for chronic obstructive pulmonary disease. FASEB J. 2015:29:2912-29.

46. Ito S, Araya J, Kurita Y, Kobayashi K, Takasaka N, Yoshida M, Hara H, Minagawa S, Wakui H, Fujii S, et al. PARK2-mediated mitophagy is involved in regulation of $\mathrm{HBEC}$ senescence in COPD pathogenesis. Autophagy. 2015;11:547-59.

47. Wang P, Lindsay J, Owens TW, Mularczyk EJ, Warwood S, Foster F, Streuli CH, Brennan K, Gilmore AP. Phosphorylation of the proapoptotic BH3-only protein bid primes mitochondria for apoptosis during mitotic arrest. Cell Rep. 2014;7:661-71.

48. Garcia-Perez C, Roy SS, Naghdi S, Lin X, Davies E, Hajnoczky G. Bid-induced mitochondrial membrane permeabilization waves propagated by local reactive oxygen species (ROS) signaling. Proc Natl Acad Sci U S A. 2012;109:4497-502

49. Kang MJ, Shadel GS. A mitochondrial perspective of chronic obstructive pulmonary disease pathogenesis. Tuberc Respir Dis (Seoul). 2016;79:207-13.

50. Park JW, Ryter SW, Choi AM. Functional significance of apoptosis in chronic obstructive pulmonary disease. COPD. 2007:4:347-53.

51. Zhu B, Chen C, Xue G, Lei X, Li J, Moyzis RK, Dong Q, Lin C. The GABRB1 gene is associated with thalamus volume and modulates the association between thalamus volume and intelligence. Neuroimage. 2014;102(Pt 2):756-63.

52. Jin N, Kolliputi N, Gou D, Weng T, Liu L. A novel function of ionotropic gamma-aminobutyric acid receptors involving alveolar fluid homeostasis. J Biol Chem. 2006:281:36012-20.

53. Wang G, Wang R, Ferris B, Salit J, Strulovici-Barel Y, Hackett NR, Crystal RG. Smoking-mediated up-regulation of GAD67 expression in the human airway epithelium. Respir Res. 2010;11:150. 
54. Satta R, Maloku E, Zhubi A, Pibiri F, Hajos M, Costa E, Guidotti A. Nicotine decreases DNA methyltransferase 1 expression and glutamic acid decarboxylase 67 promoter methylation in GABAergic interneurons. Proc Natl Acad Sci U S A. 2008;105:16356-61.

55. Shenker NS, Polidoro S, van Veldhoven K, Sacerdote C, Ricceri F, Birrell MA, Belvisi MG, Brown R, Vineis P, Flanagan JM. Epigenome-wide association study in the European Prospective Investigation into Cancer and Nutrition (EPIC-Turin) identifies novel genetic loci associated with smoking. Hum Mol Genet. 2013;22:843-51.

56. Ambatipudi S, Cuenin C, Hernandez-Vargas H, Ghantous A, Le Calvez-Kelm F, Kaaks R, Barrdahl M, Boeing H, Aleksandrova K, Trichopoulou A, et al. Tobacco smoking-associated genome-wide DNA methylation changes in the EPIC study. Epigenomics. 2016;8:599-618.

57. Zhang Y, Breitling LP, Balavarca Y, Holleczek B, Schottker B, Brenner $H$. Comparison and combination of blood DNA methylation at smokingassociated genes and at lung cancer-related genes in prediction of lung cancer mortality. Int J Cancer. 2016:139:2482-92.

58. Philibert RA, Beach SR, Lei MK, Brody GH. Changes in DNA methylation at the aryl hydrocarbon receptor repressor may be a new biomarker for smoking. Clin Epigenetics. 2013;5:19.

59. Wauters $E$, Janssens W, Vansteenkiste J, Decaluwe H, Heulens N, Thienpont B, Zhao H, Smeets D, Sagaert X, Coolen J, et al. DNA methylation profiling of non-small cell lung cancer reveals a COPD-driven immune-related signature. Thorax. 2015;70:1113-22.

60. Suzuki M, Wada H, Yoshino M, Tian L, Shigematsu H, Suzuki H, Alaa M, Tamura H, Fujiwara T, Nagato K, et al. Molecular characterization of chronic obstructive pulmonary disease-related non-small cell lung cancer through aberrant methylation and alterations of EGFR signaling. Ann Surg Oncol. 2010;17:878-88

61. Tessema M, Yingling CM, Picchi MA, Wu G, Liu Y, Weissfeld JL, Siegfried JM, Tesfaigzi Y, Belinsky SA. Epigenetic repression of CCDC37 and MAP1B links chronic obstructive pulmonary disease to lung cancer. J Thorac Oncol. 2015:10:1181-8.

\section{Submit your next manuscript to BioMed Central and we will help you at every step:}

- We accept pre-submission inquiries

- Our selector tool helps you to find the most relevant journal

- We provide round the clock customer support

- Convenient online submission

- Thorough peer review

- Inclusion in PubMed and all major indexing services

- Maximum visibility for your research

Submit your manuscript at www.biomedcentral.com/submit 